\title{
EN01 suppresses cancer cell ferroptosis by degrading the mRNA of iron regulatory protein 1
}

\section{Huafeng Zhang ( $\nabla$ hzhang22@ustc.edu.cn )}

University of Science and Technology of China https://orcid.org/0000-0002-6930-7989

\section{Tong Zhang}

The First Affiliated Hospital of USTC, University of Science and Technology of China, Hefei 230027, China

\section{Linchong Sun}

Hefei National Laboratory for Physical Sciences at Microscale, CAS Key Laboratory of Innate Immunity and Chronic Disease, School of Life Sciences, University of Science and Technology of China, Hefei

\section{Yijie Hao}

Hefei National Laboratory for Physical Sciences at Microscale, CAS Key Laboratory of Innate Immunity and Chronic Disease, School of Life Sciences, University of Science and Technology of China, Hefei

\section{Wenhao Ma}

Hefei National Laboratory for Physical Sciences at Microscale, CAS Key Laboratory of Innate Immunity and Chronic Disease, School of Life Sciences, University of Science and Technology of China, Hefei

\section{Shengqi Shen}

Hefei National Laboratory for Physical Sciences at Microscale, the CAS Key Laboratory of Innate Immunity and Chronic Disease, School of Life Sciences, University of Science and Technology of China

\section{Caixia Suo}

Laboratory of Cancer and Stem Cell Metabolism, School of Medicine, Institutes for Life Sciences, South China University of Technology, Guangzhou 510006, China

\section{Pinggen Zhang}

University of Science and Technology of China

\section{Ting Wang}

University of Science and Technology of China

\section{Shi-Ting Li}

Hefei National Laboratory for Physical Sciences at Microscale, the CAS Key Laboratory of Innate Immunity and Chronic Disease, School of Life Sciences, University of Science and Technology of China

\section{Zhaolin Chen}

The First Affiliated Hospital of USTC, University of Science and Technology of China

\section{Haoran Wei}

University of Science and Technology of China

\section{Ronghui Yan}

University of Science and Technology of China 


\section{Yi Zhang}

South China University of Technology

\section{Yongping Cai}

Department of Pathology, School of Medicine, Anhui Medical University

\section{Weidong Jia}

University of Science and Technology of China

\section{Fang Huang}

University of Science and Technology of China

\section{Ping Gao}

University of Science and Technology of China

\section{Article}

Keywords: a-Enolase 1, iron regulatory protein 1, cancer

Posted Date: October 2nd, 2020

DOl: https://doi.org/10.21203/rs.3.rs-79778/v1

License: (c) (i) This work is licensed under a Creative Commons Attribution 4.0 International License. Read Full License

Version of Record: A version of this preprint was published at Nature Cancer on December 9th, 2021. See the published version at https://doi.org/10.1038/s43018-021-00299-1. 


\section{ENO1 suppresses cancer cell ferroptosis by degrading the mRNA of iron}

\section{regulatory protein 1}

Tong Zhang ${ }^{1,7}$, Linchong Sun ${ }^{3,4,7}$, Yijie Hao ${ }^{1}$, Wenhao $\mathrm{Ma}^{1}$, Shengqi Shen ${ }^{1}$, Caixia Suo ${ }^{3}$, Pinggen Zhang ${ }^{1}$, Ting Wang ${ }^{1}$, Shi-Ting $\mathrm{Li}^{1}$, Zhaolin $\mathrm{Chen}^{2}$, Haoran Wei ${ }^{1}$, Ronghui Yan ${ }^{1}$, Yi Zhang ${ }^{3}$, Yongping Cai ${ }^{6}$, Weidong $\mathrm{Jia}^{2}$, Fang Huang ${ }^{5}$, Ping Gao ${ }^{3,4 *}$, Huafeng Zhang ${ }^{1,2 *}$

${ }^{1}$ Hefei National Laboratory for Physical Sciences at Microscale, the CAS Key Laboratory of Innate Immunity and Chronic Disease, School of Basic Medical Sciences, Division of Life Sciences and Medicine, University of Science and Technology of China, Hefei, China.

${ }^{2}$ The First Affiliated Hospital of USTC, University of Science and Technology of China, Hefei 230027, China.

${ }^{3}$ Guangzhou First People's Hospital, School of Medicine, Institutes for Life Sciences, South China University of Technology, Guangzhou, China.

${ }^{4}$ School of Biomedical Sciences and Engineering, Guangzhou International Campus, South China University of Technology, Guangzhou, China.

${ }^{5}$ CAS Key Laboratory of Crust-Mantle Materials and Environments, School of Earth and Space Sciences, University of Science and Technology of China, Hefei 230026, China.

${ }^{6}$ Department of Pathology, School of Medicine, Anhui Medical University, Hefei, China.

${ }^{7}$ These authors contributed equally: Tong Zhang, Linchong Sun.

*e-mail: hzhang22@ustc.edu.cn, pgao2@ustc.edu.cn

$\alpha$-Enolase 1 (ENO1) is a critical glycolytic enzyme whose aberrant expression drives the pathogenesis of various cancers. ENO1 has been indicated to have additional roles beyond its conventional metabolic activity, but the underlying mechanisms and biological consequences remain elusive. Here, we show that ENO1 suppresses iron regulatory protein 1 (IRP1) expression to regulate iron homeostasis and survival of hepatocellular carcinoma (HCC) cells. Mechanistically, we unprecedentedly uncover that ENO1, as an RNA-binding protein, recruits CNOT6 to accelerate the mRNA decay of IRP1 in cancer cells, leading to inhibition of mtioferin-1 (Mfrn1) expression and subsequent repression of mitochondrial iron-induced ferroptosis. Moreover, through in vitro and in vivo experiments and clinical sample analysis, we identified IRP1 and Mfrn1 as tumor suppressors by inducing ferroptosis in HCC cells. 
Taken together, this study establishes a novel role for the ENO1/IRP1/Mfrn1 pathway in the pathogenesis of HCC and reveals a previously unknown connection between the ENO1/IRP1/Mfrn1 pathway and ferroptosis, suggesting a potential innovative cancer therapy.

Cancer cells undergo extensive metabolic reprogramming including the typical aerobic glycolysis or the Warburg effect, which involves the switch of a multitude of metabolic enzymes to support the diversion of metabolites that facilitate glycolytic fermentation for energy metabolism even under ambient oxygen conditions ${ }^{1-4}$. Compelling literatures have recently revealed that many glycolytic enzymes have moonlighting activities in regulating gene expression ${ }^{5-7}$. For example, GAPDH, which converts glyceraldehyde-3-phosphate to D-glycerate-1, 3-bisphosphate, has been unexpectedly found as the RNA-binding protein that regulates effector T cell function by binding to the IFN- $\gamma$ mRNA to repress protein translation ${ }^{8}$. PKM2 also functions as a protein kinase and phosphorylates a variety of protein substrates to regulate gene expression, mitosis, cytokinesis and exosome secretion ${ }^{7,9}$. ENO1 is a key glycolytic enzyme that catalyzes the dehydration of 2-phosphoglycerate to phosphoenolpyruvate. ENO1 is associated with tumor progression and is considered as a potential diagnostic marker for many cancers ${ }^{10-12}$. Besides its glycolytic function, ENO1 acts as a strong plasminogen receptor on the surface of hematopoietic cells such as T cells, B cells, monocytes, neuronal cells and endothelial cells ${ }^{13,14}$. Furthermore, ENO1 binds to F-actin and tubulin and could localize at centrosomes, indicative of its potential roles in cell cycle regulation ${ }^{15}$, 16. More recently, ENO1 has been identified as a candidate of an RNA-binding protein from an "interactome capture" study ${ }^{17}$, nevertheless, its relevance to cancer development and the underlying mechanisms are still elusive.

Intriguingly, ENO1 belongs to a member of the RNA degradosome in Escherichia coli and facilitates RNA degradation ${ }^{18}$, however, whether this function is conserved in eukaryotic cells remains to be explored. Meanwhile, the functions of many genes are highly similar in eukaryotes and prokaryotes. For example, the basic machinery of DNA replication has high conservation in prokaryotes and eukaryotes ${ }^{19}$, despite the more complicated regulatory strategies in eukaryotic cells. Since ENO1 plays important roles in the RNA degradation in prokaryotes, we endeavor to investigate whether ENO1 promotes mRNA degradation in the eukaryotic cells. 
Iron is an essential element for metabolic regulation, and its roles in regulation of cell biological events have been multifaceted. For example, iron also participates in potentially deleterious free radical reactions that damage lipids, proteins and DNA. Thus, iron homeostasis is maintained precisely in cells by iron transport, storage and regulatory proteins ${ }^{20}$. Both the beneficial and deleterious effects of iron have been reported during cancer development ${ }^{21-25}$. Ferroptosis is a form of regulated cell death characterized by the iron-dependent accumulation of lipid hydroperoxides ${ }^{23}$, ${ }^{26}$, which is morphologically, genetically and biochemically distinct from other forms of cell death, such as apoptosis, necroptosis and autophagy ${ }^{26}$. Dysregulation of ferroptosis is associated with various human diseases, such as neurodegeneration, ischaemia-reperfusion injury (IRI) and cancer ${ }^{23}$, 27-31. However, the regulatory mechanisms of ferroptosis in tumor biology remain largely unknown. Since iron is a crucial factor required for the accumulation of lipid peroxides, ultimately leading to ferroptosis $^{22}$, it is essential to elucidate the detailed mechanisms by which cancer cells regulate iron homeostasis, metabolic reprogramming and ferroptosis to support cancer progression.

In this study, we seek to identify novel moonlighting functions for the glycolytic enzyme ENO1. We unprecedentedly find that ENO1, as an RNA-binding protein, conserves its RNA degrading function from prokaryotes to eukaryotes. We uncover that ENO1 promotes mRNA degradation of IRPI to maintain iron homeostasis in cancer cells, protecting cancer cells from ferroptotic cell death. We established a novel connection between the ENO1/IRP1/Mfrn1 pathway and ferroptosis, which is highly relevant to HCC progression. This knowledge will benefit the potential development of therapeutic strategies involving the induction of ferroptosis in liver cancer.

\section{Results}

ENO1 degrades IRP1 mRNA as an RNA-binding protein. To explore whether ENO1 has roles beyond its known glycolytic function, we asked whether ENO1 has conserved roles in RNA degradation in eukaryotes. We first performed RNA-Seq analysis in PLC cells with ENO1 knockdown. We observed that many genes were regulated by ENO1 (Fig. 1a). Combining RNA-seq 
data with previous ENO1 CLIP-Seq data ${ }^{17}$, 17 genes were found to be negatively regulated and bound by ENO1 (Fig. 1b). Quantitative reverse transcriptase PCR (qPCR) confirmed that ANXA1, IRP1, KANK1, CAT, BIN1, and MYO6 among 17 genes were strongly suppressed by ENO1 at the mRNA levels (Fig. 1c). We further investigated the expression of the 17 genes in paired clinical hepatocellular carcinoma (HCC) lesions and adjacent noncancerous tissues (Fig. 1d and Extended Data Fig. 1a). The results showed that iron regulatory protein 1 (IRPl) mRNA was significantly decreased in the HCC lesions (Fig. 1d).

RNA immunoprecipitation (RIP) showed that ENO1 bound to IRPI mRNA in PLC cells (Fig. 1e). ENO1 over-expression and knockdown systems consistently showed that ENO1 inhibited IRP1 expression in PLC cells (Fig. 1f). Similar results were also observed in two additional HCC cell lines, HepG2 and Hep3B cells (Extended Data Fig. 1b). Interestingly, IRP1 mRNA was significantly degraded in ENO1 overexpressing cells in the presence of actinomycin D, a transcription inhibitor, indicating that ENO1 negatively regulated IRP1 expression by disrupting its mRNA stability (Fig. 1g). RNA decay analysis using nascent RNA in living cells labelled with ethynyl uridine (EU) revealed that ENO1 accelerated IRPI mRNA degradation (Fig. 1h). In addition, ENO1 promoted the RNA decay of the other genes mentioned in Fig.1c (Extended Data Fig. 1c), demonstrating that ENO1 extensively regulates gene expression by accelerating RNA degradation.

Recent studies have shown that the dual DNA- and RNA-binding capacity of a growing body of proteins may play an important role in modulating gene expression ${ }^{32}$. ENO1 has a DNA-binding domain $(\mathrm{DBD})^{33}$, and different deletion mutations of the DNA-binding region of ENO1 attenuated the inhibitory effect of ENO1 on both IRPI mRNA and protein expression, as well as its ability to bind to IRP1 mRNA (Fig. 1i,j). Our results further showed that $\mathrm{ENO} 1^{\mathrm{S} 40 \mathrm{~A}}$ or $\mathrm{ENO} 1^{\mathrm{D} 245 \mathrm{R}}$, the catalytically dead mutants of ENO1 ${ }^{34}$, exhibited a similar IRPI mRNA binding capacity as the wild-type ENO1 (Fig. 1j). Moreover, MBP1, an alternative translation product of ENO1 mRNA, that contains the DNA-binding domain of ENO1 ${ }^{35}$, also inhibited IRP1 via its DNA-binding domain (Fig. 1k). Taken together, we thus conclude that ENO1 binds to IRPI mRNA via its DNA-binding domain. On the other hand, a dual-luciferase reporter assay demonstrated that the 5' untranslated 
region (5'UTR) of IRP1 mRNA was important for its association with ENO1 protein (Fig. 11). More interestingly, consistent with a previous report that $\mathrm{CpG}$ dinucleotides located within the 5'UTR accelerated mRNA decay ${ }^{36}$, deletion of the CpG-rich region in the IRP1 5'UTR abolished the inhibitory effect of ENO1 on IRP1, indicating that the CpG-rich region of the IRPI 5'UTR is important for its association with ENO1 (Fig. 1m). In conclusion, ENO1, as an RNA-binding protein, binds to the CpG-rich region of IRP1 5'UTR via its DNA-binding domain (DBD) and ultimately promotes the degradation of IRPI mRNA (Fig. 1n).

ENO1 recruits CNOT6 to degrade IRP1 mRNA. We then explored the mechanism of ENO1-mediated IRP1 degradation. Mass spectrometry (MS) data from a previous report showed that ENO1 interacted with the CCR4-NOT deadenylase complex, which plays a central role in mRNA regulation by catalyzing the removal of mRNA poly (A) tails ${ }^{37}$. Our analysis of the poly (A) tail length showed that ENO1 promoted the deadenylation of IRP1 mRNA (Fig. 2a), suggesting the involvement of CCR4-NOT deadenylase complex in ENO1 mediated IRP1 mRNA degradation. To further identify the factor(s) involved in ENO1-mediated RNA degradation, we screened the components of the CCR4-NOT complex that could interact with ENO1 using co-immunoprecipitation (Co-IP) assays. As a result, CNOT3, CNOT6 and CNOT10 were found to interact with ENO1 (Fig. 2b). However, GST pull-down experiments showed that only CNOT6, rather than CNOT3 or CNOT10, interacted directly with ENO1 (Fig. 2c and Extended Data Fig. 2a). This was further confirmed by co-immunoprecipitation experiments using Flag-ENO1 combined with HA-CNOT6 and vice versa (Fig. 2d and Extended Data Fig. 2b). Importantly, CNOT6 significantly suppressed IRP1 at both the mRNA and protein levels (Fig. 2e).

Further Co-IP experiments revealed that the leucine repeat domain (LR) of CNOT6 mediated its interaction with ENO1 (Fig. 2f). On the other hand, the C-terminal region of the ENO1 protein was important for its interaction with CNOT6 (Fig. 2g,h and Extended Data Fig. 2c). This was further confirmed by co-immunoprecipitation experiments showing that the C-terminal region of the ENO1 interacted with the leucine repeat domain (LR) of CNOT6 (Fig. 2i and Extended Data Fig. 2d). Our data also demonstrated that the interaction between ENO1 and CNOT6 was independent of the presence of RNA (Extended Data Fig. 2e). More importantly, suppression of ENO1 abolished the 
inhibitory effect of CNOT6 on IRP1 expression, demonstrating that CNOT6 relied on ENO1 to inhibit IRP1 expression (Fig. 2j). In conclusion, ENO1 binds to the 5'UTR of IRP1 mRNA via its DNA-binding domain and recruits CNOT6 via its C terminal domain, whereby the nuclease (NU) domain of CNOT6 promotes the deadenylation of the IRP1 3'UTR (Fig. 2k).

ENO1 promotes liver cancer by inhibiting the IRP1/Mfrn1 axis. Consistent with a previous report that IRP1 barely affects the proliferation of cultured cancer cells ${ }^{38}$, we observed that IRP1 marginally inhibited the proliferation of PLC cells (Fig. 3a). However, given that IRP1 is an important factor involved in iron homeostasis, we manipulated the iron concentration in cultured medium and found that, in the presence of iron, IRP1 dramatically inhibited cell proliferation (Fig. 3a). Meanwhile, knockdown of IRP1 promoted cell proliferation (Extended Data Fig. 3a). Notably, same concentration of iron used in the above experiments $(200 \mu \mathrm{M})$ alone showed no obvious effect on cell numbers (Extended Data Fig. 3b). Overexpression of IRP1 strongly impaired growth of tumor xenografts in mice (Fig. 3b and Extended Data Fig. 3c). More importantly, we found that the serum iron concentration of clinical liver cancer patients was significantly higher than that of healthy subjects (Fig. 3c), which is consistent with the report that increased iron levels in the body are associated with an increased risk of cancer $^{24}$ and that excess iron in the liver was more frequently observed in HCC patients than in healthy people ${ }^{39,40}$. Altogether, our results suggest that IRP1 sensitizes liver tumor cells to iron and serves as a tumor suppressor.

IRP1 plays an important role in mitochondrial iron homeostasis and is essential for liver physiology and function ${ }^{41,42}$. However, the relationship between IRP1 and mitochondria is poorly understood in tumors. Therefore, we performed real-time PCR to analyze the expression of mitochondrial iron-sulfur cluster synthesis-related genes (Fig. 3d). Interestingly, Mfrn1, a mitochondrial channel protein, which carries iron from the cytoplasm into the mitochondria ${ }^{43}$, was significantly downregulated by shIRP1 (Fig. 3d). Our data further showed that IRP1 enhanced Mfrn1 expression at both mRNA and protein levels (Fig. 3e and Extended Data Fig. 3d). Similar results were observed in Hep3B and HepG2 cells (Extended Data Fig. 3e). IRP1, together with a 4Fe-4S cluster, functions as a cytosolic aconitase by catalyzing the conversion of citrate to isocitrate. Our data showed that IRP1 ${ }^{\mathrm{C} 437 \mathrm{~S}}$, the enzymatically inactive mutation of IRP1 ${ }^{38}$, exhibited similar effect as the 
wild-type IRP1 on Mfrn1 expression and cell proliferation (Extended Data Fig. 3f), suggesting that the regulation of Mfrn1 by IRP1 was independent of its enzymatic activity. Rather, our data suggested that IRP1 activated Mfrn1 transcription via CREB (Extended Data Fig. 3g,h). Intriguingly, Mfrn1 inhibited cell growth, especially in the presence of iron (Fig. 3f). Importantly, ENO1 suppressed Mfrn1 expression at both the mRNA and protein levels (Fig. 3g and Extended Data Fig. 3i), which was abolished by IRP1 (Fig. 3h and Extended Data Fig. 3j). Consistent with Figure 1i, our results further showed that ENO1 $1^{\mathrm{S} 40 \mathrm{~A}}$ and $\mathrm{ENO} 1^{\mathrm{D} 245 \mathrm{R}}$, the catalytically dead mutants of ENO1, exhibited a similar effect as the wild-type ENO1 on the inhibition of IRP1 or Mfrn1 (Extended Data Fig. 3k). Collectively, these data demonstrate that ENO1, as an RNA-binding protein, suppresses Mfrn1 expression in an IRP1-dependent manner.

Cell proliferation and mouse xenograft experiments further revealed that overexpression of IRP1 or Mfrn1 diminished ENO1-mediated promotion of cell proliferation and tumor growth (Fig. 3i and Extended Data Fig. 4a-c). Moreover, overexpression of Mfrn1 abolished shIRP1-enhanced cell proliferation and tumor growth (Fig. 3j and Extended Data Fig. 4d,e). Collectively, these data document that the IRP1/Mfrn1 axis is involved in ENO1-regulated cell proliferation and tumor growth both in vitro and in vivo.

Neither IRP1 nor Mfrn1 has previously been implicated in the pathogenesis of liver cancer. By employing a spontaneous mouse model of $\mathrm{HCC}^{44}$, we injected YAP-5SA plasmids alone or in combination with IRP1 or Mfrn1 plasmid into the mouse livers and observed that both IRP1 and Mfrn1 retarded tumor growth in YAP-5SA-induced liver cancer (Fig. 3k). Since $M f r n I^{-/-}$mice were embryonic lethal ${ }^{45}$, we generated $M f r n I^{+/-}$mice to explore the effect on HCC tumorigenesis (Extended Data Fig. 4f). In the YAP-5SA-induced HCC model, we observed a significantly increased incidence of liver cancer and enhanced tumor growth in the $\mathrm{MfrnI}^{+/-}$mice compared to the WT mice (Fig. 31). Western blot analysis confirmed the downregulation of Mfrn1 in tumor tissues from the MfrnI ${ }^{+/-}$mice (Extended Data Fig. 4g). Thus, our results document that IRP1 and Mfrn1 serve as tumor suppressors in HCC.

\section{The ENO1/IRP1/Mfrn1 axis regulates ferroptosis by modulating mitochondrial iron}


homeostasis. Mfrn1 was known to promote the transfer of iron from the cytoplasm to mitochondria $^{43}$.We observed that ENO1 decreased while IRP1 and Mfrn1 increased mitochondrial iron accumulation (Fig. 4a). Moreover, IRP1-mediated accumulation of mitochondrial iron was diminished by shMfrn1, and ENO1-mediated reduction of mitochondrial iron was reversed by either IRP1 or Mfrn1 overexpression (Fig. 4b,c). Many iron-sulfur clusters are involved in the transmission of electrons in mitochondrial electron transport chains, and our data also showed that the ENO1/IRP1/Mfrn1 axis regulated mitochondrial complex I and II activities and mitochondrial respiration (Extended Data Fig. 5a-g). Consistent with a previous report that accelerated mitochondrial respiration led to excess mitochondrial ROS accumulation in cancer cells ${ }^{46}$, we observed that both IRP1 and Mfrn1 induced mitochondrial ROS generation and led to cell death in the presence of iron (Fig. 4d and Extended Data Fig. 6a), and Mfrn1 knockdown abolished IRP1-mediated mitochondrial ROS accumulation (Extended Data Fig. 6b).

Our western blot analysis revealed that IRP1 and Mfrn1 failed to induce the cleavage of caspase-3 or PARP1 or the accumulation of LC3-II protein (Extended Data Fig. 6c), suggesting that neither apoptosis nor autophagy was involved in IRP1/Mfrn1 axis-induced cell death. Recent studies have shown that mitochondrial function and iron metabolic disorders lead to ferroptosis, an

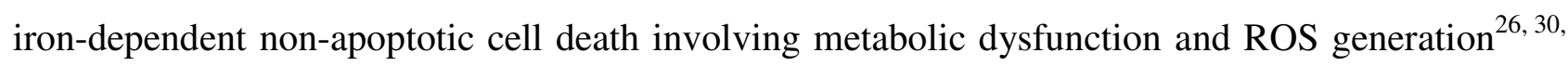
${ }^{47-51}$. We thus studied the relationship between the IRP1/Mfrn1 axis and ferroptosis. First, we treated PLC cells overexpressing IRP1 or Mfrn1 with various inhibitors, including ferrostatin-1 (Ferr-1, ferroptosis inhibitor), deferiprone (mitochondrial iron chelator) ${ }^{52}$, Z-VAD-FMK (apoptosis inhibitor), necrostatin-1 (Nec-1, necroptosis inhibitor), and 3-methyladenine (3-MA, autophagy inhibitor). As a result, Ferr-1 and iron-related deferiprone substantially rescued the cell death induced by IRP1 or Mfrn1 in the presence of iron (Fig. 4e,f), and also counteracted the inhibitory effect of IRP1 or Mfrn1 on cell proliferation in the presence of iron (Extended Data Fig. 6d,e), suggesting that ferroptosis is involved in IRP1- or Mfrn1-induced cell death. Furthermore, we observed that overexpression of IRP1 or Mfrn1 increased iron-induced lipid peroxidation (Fig. 4g) and LDH release (Extended Data Fig. 6f), two markers of ferroptosis. Transmission electron microscopy analysis revealed smaller shrunken mitochondria in the IRP1- or Mfrn1-overexpressed PLC cells than the control cells in the presence of iron, which further confirmed the morphological 
signature of ferroptotic cells (Fig. 4h). Taken together, these data demonstrate that IRP1 and Mfrn1 activate ferroptosis in liver cancer cells.

Further detection of cell death and lipid peroxidation by flow cytometry showed that IRP1-induced cell death and lipid ROS generation were strongly abolished by suppression of Mfrn1 (Fig. 4i,j). More importantly, suppression of ENO1 led to increased cell death and enhanced lipid ROS generation, which was attenuated significantly by further knockdown of IRP1 or Mfrn1 (Fig. 4k-m). Collectively, these data reveal a previously unappreciated link between the ENO1/IRP1/Mfrn1 axis and ferroptosis in liver cancer cells.

IRP1 and Mfrn1 deficiency predicts poor clinical prognosis. Analysis of cell proliferation in vitro and liver cancer growth in vivo strongly indicated that IRP1 and Mfrn1 have tumor-suppressive effects. To further investigate the pathological significance of our findings, we examined IRPI and Mfrnl mRNA expression in 17 paired clinical human HCC lesions and adjacent noncancerous tissue samples. The results showed that the IRPI and MfrnI mRNA levels were significantly decreased in the HCC lesions compared to the adjacent noncancerous tissue (Fig. 1d and Extended Data Fig. 7a). Consistently, western blot analysis revealed decreased IRP1 and Mfrn1 protein levels but increased ENO1 protein levels in human HCC tissues compared to the corresponding adjacent noncancerous tissues (Fig. 5a). More interestingly, similar results were observed in the YAP-5SA-induced mouse HCC samples (Fig. 5b).

Next, immunohistochemistry (IHC) was employed to analyze ENO1, IRP1 or Mfrn1 expression in a retrospective cohort of 135 clinicopathologically characterized HCC cases, including 14 cases of stage I (10.4\%), 75 cases of stage II (55.6\%), 30 cases of stage III (22.2\%), and 16 cases of stage IV(11.8\%) liver cancer cases, based on the TNM staging (Table S1). IHC results demonstrated that IRP1 or Mfrn1 protein was abundantly expressed in normal liver tissues and weakly expressed in HCC tissues, while ENO1 showed the opposite trend (Fig. 5c). Quantitative analysis of the IHC results revealed that IRP1 or Mfrn1 expression in clinical stage I-IV primary tumors were significantly decreased (Fig. 5d,e), but ENO1 expression was significantly increased compared to that in the normal liver tissues (Extended Data Fig. 7b). Furthermore, IRP1 and Mfrn1 were 
drastically downregulated in late-stage HCC (stages III and IV) compared to early-stage HCC (stages I and II), while ENO1 showed the opposite trend (Tables S2, S3 and S4). Moreover, Spearman analysis revealed correlations between the ENO1/IRP1/Mfrn1 axis and patient clinicopathological characteristics, including survival time, vital status, clinical stage, and tumor size (Tables S5, S6 and S7), further suggesting a strong association of ENO1, IRP1 or Mfrn1 expression with HCC clinical staging and patient survival. Finally, the Kaplan-Meier test indicated that patients expressing high IRP1 or Mfrn1 in their HCC lesions survived much longer than those with low IRP1 or Mfrn1 expression (Fig. 5f,g), suggesting that IRP1 or Mfrn1 proteins represented promising prognostic biomarkers in HCC, while ENO1 indicated the opposite outcome (Extended Data Fig. 7c). Further IHC analysis using serial sections of the same HCC tissues confirmed the correlation of high ENO1 and low IRP1 or Mfrn1 (Fig. 5h). Moreover, our IHC data showed markedly decreased expression of IRP1 or Mfrn1 in carcinoma tissues compared with the para-carcinoma tissues (Extended Data Fig. 7d,e). Thus, these results demonstrate that the ENO1/IRP1/Mfrn1 axis is correlated with human HCC and predicts clinical prognosis.

\section{Discussion}

Enhanced glycolysis is a hallmark of cancer cells and is facilitated by oncogenes such as MYC, HIF1, Ras and AKT, which eventually leads to aberrantly induced expression of glycolytic enzymes in tumors ${ }^{1,53}$. Intriguingly, numerous metabolic enzymes have emerged as RNA-binding proteins with roles beyond catalyzing glycolysis ${ }^{5}$. ENO1 is known to accelerate cancer progression as a glycolytic enzyme ${ }^{12}$. However, recent findings on ENO1 indicate that it may serve as an RNA-binding protein with moonlighting functions ${ }^{17,54,55}$. ENO1 is a member of the RNA degradosome in prokaryotes ${ }^{18}$. This prompted us to speculate that ENO1 might mediate mRNA decay to promote cancer development. We demonstrate here that ENO1 accelerates mRNA degradation of many genes including IRPI (Fig. 1g,h and Extended Data Fig. 1c). We further uncover that CNOT6 is a key factor driving ENO1-mediated IRPI mRNA degradation (Fig. 2b,c,j). ENO1 serves as a sensor linking the energetic state to mRNA degradation in prokaryotes ${ }^{18}$. However, in eukaryotes, we find here that ENO1 acts as an RNA-binding protein to degrade RNAs by recruiting RNA degradation factor CNOT6, demonstrating the conservation and progression of 
functional complexity of ENO1 during evolution. Thus, our findings identify a new function of ENO1 that mediates mRNA degradation in mammalian cells and have enriched our knowledge on the role of ENO1 as a novel moonlighting enzyme.

Iron metabolism is a fascinating research area because of the metal's dualities in multiple biological processes $^{56}$. The iron biologists currently focus on understanding iron homeostasis and dys-homeostasis in malignant tumors through which enables the identification of new therapeutic approaches for cancer ${ }^{21,22,24}$. Earlier studies suggest that iron metabolism promotes tumor initiation and progression ${ }^{24}$. Nonetheless, recent studies demonstrate that excessive iron paradoxically has a deleterious effect on tumor, leading to ferroptosis in particular ${ }^{21,23,25,57,58}$. Ferroptosis is a unique type of cell death resulting from metabolic dysfunction involving iron metabolism, lipid metabolism, oxidant metabolism and energy metabolism ${ }^{22}$. The mechanisms underlying ferroptosis during cancer development are largely elusive. In our study, we found that IRP1 was suppressed by ENO1 via a novel RNA degradation mechanism, which led to decreased Mfrn1 expression. Significantly, we provide a novel mechanism that IRP1 promotes ferroptosis by modulating Mfrn1-induced mitochondrial iron enrichment (Fig. 4d-j). Although a previous study has shown that IRP1 may play a role in ferroptosis in mouse embryonic fibroblasts (MEFs) by RNAi screening ${ }^{59}$, we demonstrated for the first time that suppression of IRP1 protected cancer cell from ferroptotic cell death. We also demonstrated for the first time the tumor suppressive function of Mfrn1 through iron homeostasis, which is crucial to elicit ferroptosis. Other mechanisms involved in Mfrn1-mediated tumor growth inhibition merits further exploration in future studies. We observed that addition of iron dramatically enhanced the sensitivity of liver cancer cells to oxidative stress, which is consistent with the previous studies employing iron as the ferroptosis inducer ${ }^{60-62}$. More interestingly, we find that the serum iron concentrations of liver cancer patients were significantly higher than that of healthy individuals (Fig. 3c), indicating that iron metabolism is closely related to the liver cancer progression.

Analyzing the clinical HCC samples and mouse tumor tissues, we find that IRP1 and Mfrn1 proteins were significantly decreased but ENO1 protein was significantly increased in the HCC lesions compared to the adjacent noncancerous tissue (Fig. 5a,b). Furthermore, the IHC experiments 
reveal that IRP1 or Mfrn1 expression in clinical primary tumors was significantly decreased, but ENO1 expression was significantly increased compared to that in the normal liver tissues (Fig. 5c-e and Extended Data Fig. 7b). These results suggest the ENO1/IRP1/Mfrn1 axis is highly relevant to human $\mathrm{HCC}$ development and may represent reliable $\mathrm{HCC}$ prognostic indicator. Hence, we uncovered in this study an unexpected role for ENO1 in promoting IRPI mRNA degradation via CNOT6 in cancer cells, reminiscent of a mechanism in prokaryotic cells. Importantly, we identified a novel ENO1/IRP1/Mfrn1 signaling axis that is critical for regulating ferroptosis and survival of cancer cells (Fig. 6). Furthermore, we unprecedentedly demonstrated that IRP1 and Mfrn1 function as tumor suppressors to predict the clinical prognosis of HCC. Our results shed light on the importance of the mechanisms counteracting ferroptosis in HCC progression and may serve to invite more mechanistic studies to identify innovative therapeutic approaches for liver cancer patients.

\section{Methods}

Cell culture and reagents. Human PLC, HepG2, Hep3B, HEK293 and HEK293T cells were cultured in DMEM medium containing $25 \mathrm{mM}$ glucose, $4 \mathrm{mM}$ L-glutamine and $1 \mathrm{mM}$ pyruvate (12800, Gibco). The media were supplemented with $1 \%$ penicillin-streptomycin and $10 \%$ FBS. actinomycin D (HY-17559, MCE), carboxy-H2DCFDA (C400, Invitrogen), MitoSOX (M36008, Invitrogen), BODIPY 581/591 C11 (D3861, Invitrogen), NADH (10107735001, Roche), succinate (S9637, Sigma), ubiquinone (D7911, Sigma), rotenone (R8875, Sigma), 2,6-dichlorophenolindophenol (D1878, Sigma), antimycin A (A8674, Sigma), ferrostatin-1 (HY-100579, MCE), deferiprone (HY-B0568, MCE), neccrostatin-1 (S8037, Selleck), 3-methyladenine (HY-19312 MCE), Z-VAD-FMK (HY-16658B, MCE), propidium iodine (PI) (P4864, Sigma-Aldrich), Oligo (dT) ${ }_{12-18}$ Primer (18418012, Invitrogen), SuperScript ${ }^{\mathrm{TM}}$ II Reverse Transcriptase (18064022, Thermo), and Iron (II) sulfate heptahydrate (F8263, Sigma) were obtained.

Plasmids and Established Stable Cells. All shRNAs in the PLKO vector against ENO1, IRP1, 
Mfrn1 and CREB1 were obtained commercially (Sigma-Aldrich). shRNA targeting sequences are listed in Table S8. ENO1 and its mutants, IRP1, Mfrn1 and CNOT6 were subcloned into the pSin-3 3 Flag or pSin-HA empty vector. They were co-transfected with plasmids encoding VSVG and $\triangle 8.9$ into HEK293T packaging cells using PEI (Polysciences). PLC, Hep3B or HepG2 cells were infected with the produced lentivirus in the presence of polybrene and selected with $0.5 \mu \mathrm{g} / \mathrm{ml}$ puromycin to establish stable cells.

Immunoblotting. Cells were lysed with RIPA (50 mM Tris- $\mathrm{HCl}, \mathrm{pH} 8.0,150 \mathrm{mM} \mathrm{NaCl}, 5 \mathrm{mM}$ EDTA, $0.1 \%$ SDS and 1\% NP-40) buffer and equal amounts of protein in the lysates were boiled and fractionated by $6 \%-12 \%$ SDS-PAGE. Primary antibodies against the following proteins were used: ENO1 (11204-1-AP, Proteintech), IRP1 (D261223, Sangon Biotech), Mfrn1 (26469-1-AP, Proteintech), CNOT3 (11135-1-AP, Proteintech), CNOT6 (13415S, CST), CNOT10 (15938-1-AP, Proteintech), CREB (12208-1-AP, Proteintech), CREB (phospho S133) (ab32096, Abcam), PARP1 (13371-1-AP, Proteintech), Caspase-3 (9662S, CST), LC3 (NB100-2220, Novus), Actin (66009-1-1g, Proteintech), Flag (AE024, Abclonal), HA (51064-2-AP, Proteintech), HA-HRP (2999S, CST), GST (10000-0-AP, Proteintech). HRP-conjugated anti-rabbit and anti-mouse secondary antibodies (Bio-Rad) were used. Signals were detected using Western ECL Substrate (Tanon).

qPCR. Total RNA was isolated using TRIzol followed by DNase (Ambion) treatment and reverse transcription with an iScript cDNA Synthesis Kit (Bio-Rad). qPCR was performed using SYBR Green Master Mix (Vazyme) on a Bio-Rad iCycler. Primer sequences were shown in Table S9. All samples were normalized to RPL13A.

Chromatin Immunoprecipitation Assay. The ChIP assay was performed with an EZ-ChIP kit (Millipore) following the manufacturer's instructions. Briefly, cells were sonicated by an Ultrasonic Homogenizer JY92-IIN (Scientz). DNA was immunoprecipitated by either control IgG or CREB antibody (Proteintech, 12208-1-AP), followed by quantitative real-time PCR analysis (Bio-Rad). The oligos used for this analysis are listed in Table S10. 
RNA Immunoprecipitation. For endogenous immunoprecipitation, protein A/G beads were incubated with antibody $(2 \mu \mathrm{g})$ for 2 hours at $4^{\circ} \mathrm{C}$, mixed with prepared cell lysate, and rotated $4^{\circ} \mathrm{C}$ overnight. After six washes, RNA bound to Protein A/G beads was extracted with TRIzol (Invitrogen). IgG served as a control. For exogenous immunoprecipitation, cells were lysed, mixed with anti-Flag M2 agarose slurry, and rotated $4{ }^{\circ} \mathrm{C}$ overnight. After six washes, RNA bound to anti-Flag M2 agarose slurry was competitively eluted with $3 \times$ Flag peptide, and extracted with TRIzol (Invitrogen). Empty vector (EV) served as a control.

Dual Luciferase Reporter Assay. IRP1-5'UTR, IRP1-3'UTR or IRP1-5'UTR-deletion fragments were inserted into the pGL3-basic dual-luciferase reporter vector (Promega), designated as pGL3-IRP1-5'UTR, pGL3-IRP1-3'UTR or pGL3-IRP1-5'UTR-deletion, respectively. HEK293 cells were seeded in 48-well plates. After overnight incubation, cells were co-transfected with 100 ng of firefly luciferase reporter plasmid, and $4 \mathrm{ng}$ of pSV-Renilla plasmid. Luciferase activities were measured using the Dual-Luciferase Reporter Assay System (Promega). Firefly luciferase activity was normalized to Renilla luciferase activity.

mRNA Stability Assay. PLCs were incubated in complete medium supplemented with $5 \mu \mathrm{M}$ actinomycin D for 0,3 or 6 hours. No drop in cell viability was observed at the end of the treatment. RNA was collected and mRNA levels were analyzed by qPCR. All samples were normalized with actin.

RNA Decay and Turnover Experiment. The Click-iT Nascent RNA Capture Kit (\#C10365, Thermo) was used in this experiment. The protocol was followed with slight modifications to the manufacturer as described previously ${ }^{63}$.

Immunoprecipitation. For co-immunoprecipitation, cells were lysed with low-salt IP buffer $(0.5 \%$ NP-40, 20 mM HEPES (pH 7.5), $150 \mathrm{mM} \mathrm{NaCl,} 2 \mathrm{mM}$ EDTA, $1.5 \mathrm{mM} \mathrm{MgCl}_{2}$ ) supplemented with protease inhibitor cocktail for 2 hours on ice, and centrifuged at $16,000 \mathrm{~g}$ for $10 \mathrm{~min}$ at $4^{\circ} \mathrm{C}$. The supernatants were incubated with the indicated antibody overnight at $4^{\circ} \mathrm{C}$ followed by incubation with protein $\mathrm{A} / \mathrm{G}$ conjugated beads for 1 hour. Beads were then washed with IP buffer and boiled in 
SDS-loading buffer and analyzed by western blots.

Fusion Protein Pull-down Experiment. The coding region of CNOT6 was cloned into the pSV282 vector, and cDNAs encoding CNOT3 and CNOT10 were cloned into the pET-22b vector. The cDNA encoding ENO1 was cloned into the pGEX-4T-1 vector. Proteins were produced in Escherichia coli (DE3). Purified GST fusion proteins and His-tagged proteins were used for pull-down experiments in pull-down buffer $(150 \mathrm{mM} \mathrm{NaCl}, 50 \mathrm{mM}$ Tris (pH 7.5), 0.1\% NP-40, 5 mM DTT). After incubation, the beads were pelleted and washed with pull-down buffer followed by elution of proteins and analysis by western blots.

Mitochondrial and Intracellular Reactive Oxygen Species. Mitochondrial ROS levels were measured by the MitoSOX Red fluorescence assay (Life Technologies). Cells were incubated in 5 $\mu \mathrm{M}$ MitoSOX reagent in PBS containing $5 \% \mathrm{FBS}$ at $37^{\circ} \mathrm{C}$ for $30 \mathrm{~min}$. Intracellular ROS were stained with $10 \mu \mathrm{M}$ carboxy-H2DCFDA (C400, Invitrogen) in PBS containing $5 \% \mathrm{FBS}$ at $37^{\circ} \mathrm{C}$ for 30 min. All ROS analyzed by flow cytometry (BD Bioscience) following the manufacturer's instructions.

Lipid ROS Assay. Cells were harvested and washed with PBS, and were incubated in $50 \mu \mathrm{M} \mathrm{C}-11$ BODIPY dye reagent in PBS containing $5 \% \mathrm{FBS}$ at $37^{\circ} \mathrm{C}$ for $30 \mathrm{~min}$. ROS levels were analyzed by flow cytometry (BD Bioscience) following the manufacturer's instructions.

Cell Viability. For measurement of cell viability, 5,000 cells per well were seeded in a 96-well plate 1 day before treatment. Following treatment with the appropriate drugs where indicated, each well was replaced with fresh medium containing Cell Counting Kit-8 (CCK8) reagent (Sigma). After incubation for 1 hour at $37^{\circ} \mathrm{C}$, the plate was analyzed using a microplate reader (Thermo, Multiskan Go), and the absorbance of the wells was measured at $450 \mathrm{~nm}$.

Cell Death Assay. To measure cell death, cells were seeded in a 6-well plate 1 day before treatment. Cell death was assessed by labelling with propidium iodide (PI) (P4864, Sigma-Aldrich) and was analyzed by flow cytometry (BD Biosciences). 
Measurement of Lactate Dehydrogenase (LDH) Activity. The culture medium of PLC cells was collected and LDH activity was measured using LDH-Glo ${ }^{\mathrm{TM}}$ Cytotoxicity Assay (J2380, Promega) following the manufacturer's instructions.

PAT Assays. PAT assays, analysis of poly (A) tail lengths by PCR, were performed as described previously ${ }^{64}$ with specific primers (the sequences are listed in Table S11). In brief, total RNA was incubated with poly $[\mathrm{dT}]_{12-18}$, oligo $[\mathrm{dT}]$-anchor, and T4 DNA ligase before reverse transcription reaction to generate PAT cDNA. The polyadenylation of mRNA transcripts was analyzed by PCR using a message-specific primer, oligo [dT]-anchor primer and PAT cDNA.

Active Mitochondria Extraction. Mitochondrial protein lysates were extracted from cells as previously described $^{65}$. In brief, cells were collected and homogenized in ice-cold mitochondrial isolation buffer $(10 \mathrm{mM} \mathrm{KCl}, 1.5 \mathrm{mM} \mathrm{MgCl} 2,10 \mathrm{mM}$ Tris- $\mathrm{Cl}(\mathrm{pH}$ 7.4)) supplemented with protease inhibitor cocktail (Roche, Germany). Unbroken cells and nuclei were pelleted by centrifugation at $1200 \mathrm{~g}$ for $10 \mathrm{~min}$ at $4^{\circ} \mathrm{C}$. Then the supernatants were centrifuged at $4,000 \mathrm{~g}$ for $20 \mathrm{~min}$ at $4^{\circ} \mathrm{C}$ to remove lysosomes and endoplasmic reticulum. The mitochondrial pellets were washed with isolation buffer and further centrifuged at $10,000 \mathrm{~g}$ for $15 \mathrm{~min}$ at $4{ }^{\circ} \mathrm{C}$ and were collected for subsequent experiments.

Mitochondrial Stress Test. The OCR was performed using a Seahorse Bioscience XF96 analyzer with an XF Cell Mito Stress Test Kit (103015-100, Agilent). PLC cells $\left(1 \times 10^{4}\right.$ cells/well) were seeded per well overnight in a 96-well XF cell culture microplate in growth medium. Cells were treated with oligomycin $(1 \mu \mathrm{M})$, FCCP $(1.5 \mu \mathrm{M})$ and rotenone/antimycin A $(0.5 \mu \mathrm{M})$. Data were analyzed by a Seahorse Mitochondrial Stress Test Report Generator.

Mitochondrial Iron Assays. First, complete mitochondria were extracted using the methods mentioned above. Non-heme iron was measured as described ${ }^{66}$. In brief, equal amounts of mitochondrial proteins were mixed with protein precipitation solution $(1: 11 \mathrm{~mol} / \mathrm{L} \mathrm{HCl}$ and $10 \%$ trichloroacetic acid) and heated to $95^{\circ} \mathrm{C}$ for 1 hour to release iron. Precipitated protein was removed 
by centrifugation at $4^{\circ} \mathrm{C}$ at $16,000 \mathrm{~g}$ for $10 \mathrm{~min}$. The supernatant was mixed with the equal volume of chromogen solution $(0.5 \mathrm{mM}$ ferrozine, $1.5 \mathrm{M}$ sodium acetate, $0.1 \%$ [v/v] thioglycolic acid). The absorbance was measured on a Thermo Mutiscan GO microplate reader at $562 \mathrm{~nm}$.

Mitochondrial Complex I and II Activities. Mitochondrial complex I and II activities were measured as described ${ }^{67}$. In brief, complex I activity was measured by following the decrease in absorbance due to the oxidation of NADH at $340 \mathrm{~nm}$. NADH $(0.13 \mathrm{mM})$, ubiquinone $(65 \mu \mathrm{M})$, and Antimycin A $(2 \mu \mathrm{g} / \mathrm{ml})$ were added to the assay medium (potassium phosphate, $25 \mathrm{mM}, \mathrm{pH} 7.2$ at $20^{\circ} \mathrm{C} ; \mathrm{MgCl}_{2}, 5 \mathrm{mM}$; bovine serum albumin (fraction $\mathrm{V}$ ), $2.5 \mathrm{mg} / \mathrm{ml}$ ). Mitochondria (50 $\mu \mathrm{g}$ of protein) were added, and the NADH: ubiquinone oxidoreductase activity was measured for 3-5 min. Complex II specific activity was measured by following the reduction of 2, 6-dichlorophenolindophenol at $600 \mathrm{~nm}$. Mitochondria (20 $\mu \mathrm{g}$ of protein) were preincubated in the assay medium plus succinate $(20 \mathrm{mM})$ at $30^{\circ} \mathrm{C}$ for $10 \mathrm{~min}$. Antimycin $\mathrm{A}(2 \mu \mathrm{g} / \mathrm{ml})$, rotenone $(2$ $\mu \mathrm{g} / \mathrm{ml})$, and 2, 6-dichlorophenolindophenol $(50 \mu \mathrm{M})$ were added, and a baseline rate was recorded for $3 \mathrm{~min}$. The reaction was started with ubiquinone $(65 \mu \mathrm{M})$, and the enzyme-catalyzed reduction of dichlorophenolindophenol was measured for 3-5 min.

Transmission Electron Microscopy. The PLC cells were fixed with $2.5 \%$ glutaraldehyde at $4^{\circ} \mathrm{C}$ for 12 hours and then fixed in $2 \%$ osmium tetroxide. After the samples were washed, they were stained with $1 \%$ aqueous uranyl acetate. The samples were dehydrated with sequential washes in 50, 70, 90, 95 and 100\% ethanol and immersed in Eponate 12 resin. The samples were then cut into ultrathin sections and counterstained with uranyl acetate and lead citrate. Images were acquired with a transmission electron microscope (120 kV; Tecnai G2 Spirit; FEI).

RNA-Seq Analysis. Total RNA was extracted using TRIzol Reagent (Life Technologies) following the manufacturer's instructions and assessed for the RIN number to inspect RNA integrity by an Agilent Bioanalyzer 2100. A total amount of $3 \mu \mathrm{g}$ RNA per sample was used as input material for the RNA sample preparations. Sequencing libraries were generated using the NEB Next Ultra RNA Library Prep Kit for Illumina (NEB, USA) following the manufacturer's recommendations, and index codes were added to attribute sequences to each sample. RNA was sequenced by Novogene 
(Tianjin, China) on the Illumina NovaSeq 6000 platform. Reads were aligned to the human genome hg19 with Bowtie v2.2.3. Gene expression analysis was performed using the DEGSeq R package (1.26.0). Unsupervised clustering was performed using cluster and tree views and visualized using heat maps. Enrichment pathway analysis of genes was compiled from Biocarta and Kyoto Encyclopedia of Genes and Genomes (KEGG) pathway databases by DAVID Bioinformatics Resources. Original data are available in the NCBI Gene Expression Omnibus (GEO) (accession number GSE153989).

Serum Iron Detection. The serum samples from 143 liver cancer patients and 55 healthy individuals were measured by an automatic biochemical analyzer (Siemens, ADVIA 2400). For the use of these clinical materials for research purposes, prior written informed consent from the patients, as well as study approval from the Institutional Research Ethics Committee of Anhui Provincial Hospital was obtained. An iron detection kit (Siemens, 501065) was used, and $200 \mu 1$ of serum was assayed.

Knockout Mice. The C57BL/6J mice were obtained from the animal centre of the University of Science and Technology of China. $M f r n I^{+/-}$mice were generated using CRISPR-Cas9-mediated genome editing (Mfrn1 target sequence: GAACGTGATGATGATGGGTG). All of the animals were housed under a 12 hours light-dark cycle at $22-24^{\circ} \mathrm{C}$ with unrestricted access to food and water for the duration of the experiment. All of the mouse experiments were approved by The Ethics Committee of University of Science and Technology of China. The study is compliant with all relevant ethical regulations regarding animal research.

Animal Studies. All animal studies were conducted with approval from the Animal Research Ethics Committee of the University of Science and Technology of China. For xenograft experiments, $9 \times 10^{6}$ PLC cells stably overexpressing ENO1 with or without IRP1 or Mfrn1 overexpression, or knockdown of IRP1 with or without Mfrn1 overexpression were injected subcutaneously into 5-week-old male nude mice (Charles River Laboratory Animal Company, China). The tumor volumes were measured using digital calipers every 4 days and calculated using the following equation: length $(\mathrm{mm}) \times$ width $(\mathrm{mm}) \times$ depth $(\mathrm{mm}) \times 0.52$. 
Hydrodynamic Injection. Four-week-old male ICR mice from Shanghai SLAC Laboratory Animal Company and four-week-old male C57BL/6J mice $\left(M f r n I^{+/+}\right.$or $M f r n I^{+/-}$mice) were used for injection. $50 \mu \mathrm{g}$ of Plasmids expressing human YAP-5SA alone or human YAP-5SA plus mouse-IRP1 or mouse-Mfrn1 together with $10 \mu \mathrm{g}$ of plasmids expressing PB transposase were diluted in sterile Ringer's solution in a volume equal to $10 \%$ of body weight. The mixture was injected within 5 to 7 seconds through the tail vein.

Clinical Human HCC Specimens. Snap-frozen HCC tissues and the corresponding para-cancerous tissues that were at least $2 \mathrm{~cm}$ away from the edge of the tumors were taken from $17 \mathrm{HCC}$ patients by radical HCC resection in Anhui Provincial Hospital (Hefei, China). For use of these clinical materials for research purposes, prior written informed consent from the patients, as well as study approval from the Institutional Research Ethics Committee of Anhui Provincial Hospital was obtained. Total RNA and protein were extracted from paired tissues and detected by qPCR and western blots, respectively. Formalin-fixed, paraffin-embedded primary HCC specimens obtained from 135 patients were randomly selected from the archives of Anhui Provincial Hospital. The clinical data and pathological features, such as age, gender, tumor node, tumor size, vascular invasion, serum alpha-fetoprotein (sAFP), hepatitis B surface antigen (HBsAg) and cirrhosis, were collected from patient medical records. Details of the patients are shown in Tables S1-7. Tumor clinical stages were defined according to the fifth edition of the American Joint Committee on Cancer/International Union Against Cancer tumor/lymph node metastasis/distal metastasis (TNM) classification system ${ }^{68}$.

Immunohistochemistry. IHC staining was quantitatively analyzed with the AxioVision Rel.4.6 computerized image analysis system assisted with the automatic measurement programme (Carl Zeiss, Oberkochen, Germany). Six representative staining fields of each section were analyzed to verify the MOD. The MOD data were statistically analyzed by $t$-tests to compare the average MOD difference between different groups of tissues.

Statistical Analysis. The data are presented as the mean \pm s.d. of three independent experiments or 
mean \pm s.e.m. as stated. Student's t-test is used to calculate $P$-values. Statistical significance is displayed as $* P<0.05$.

\section{Acknowledgements}

This work is supported in part by the Chinese Academy of Sciences (XDB39000000), National Key R\&D Program of China (2018YFA0107103, 2018YFA0800300, 2017YFA0205600), National Natural Science Foundation of China $(81930083,81525022,91957203,81821001)$, the Program for Guangdong Introducing Innovative and Entrepreneurial Teams (2017ZT07S054) and the Fundamental Research Funds for the Central Universities (YD2070002008). Please address all the correspondence and requests for materials to H.Z. (hzhang22@ustc.edu.cn).

\section{Author contributions}

H.Z. and P.G. conceived the study and supervised the experiments. T.Z., L.S., P.G. and H.Z. designed the experiments. T.Z., L.S., Y.H., G.W., W.M., S.S., C.S., P.Z., T.W., H.W., R.Y., Y.Z. and Y.C. performed experiments. S.S. analyzed RNA-seq data. W.J. and Z.C. provided clinical specimens. S.-T.L., Y.C., W.J. and F.H. provided constructive guidance and advice. H.Z., P.G., T.Z. and L.S. wrote the paper. All the authors read and approved the manuscript.

\section{Competing interests}

The authors declare no competing interests.

\section{References}

1. Hanahan, D. \& Weinberg, R.A. Hallmarks of Cancer: The Next Generation. Cell 144, 646-674 (2011).

2. Faubert, B., Solmonson, A. \& DeBerardinis, R.J. Metabolic reprogramming and cancer progression. Science 368, 152-+ (2020).

3. Pavlova, N.N. \& Thompson, C.B. The Emerging Hallmarks of Cancer Metabolism. Cell Metabolism 23, 27-47 (2016). 
4. Sullivan, L.B., Gui, D.Y. \& Heiden, M.G.V. Altered metabolite levels in cancer: implications for tumour biology and cancer therapy. Nature Reviews Cancer 16, 680-693 (2016).

5. Castello, A., Hentze, M.W. \& Preiss, T. Metabolic Enzymes Enjoying New Partnerships as RNA-Binding Proteins. Trends in Endocrinology and Metabolism 26, 746-757 (2015).

6. $\mathrm{Xu}, \mathrm{D}$. et al. The gluconeogenic enzyme PCK1 phosphorylates INSIG1/2 for lipogenesis. Nature 580, 530-+ (2020).

7. Yang, W. et al. Nuclear PKM2 regulates beta-catenin transactivation upon EGFR activation. Nature 480, 118-U289 (2011).

8. Chang, C.-H. et al. Posttranscriptional Control of T Cell Effector Function by Aerobic Glycolysis. Cell 153, 1239-1251 (2013).

9. Lu, Z. \& Hunter, T. Metabolic Kinases Moonlighting as Protein Kinases. Trends in Biochemical Sciences 43, 301-310 (2018).

10. Cappello, P. et al. Vaccination With ENO1 DNA Prolongs Survival of Genetically Engineered Mice With Pancreatic Cancer. Gastroenterology 144, 1098-1106 (2013).

11. Chang, G.-C. et al. Identification of alpha-enolase as an autoantigen in lung cancer: Its overexpression is associated with clinical outcomes. Clinical Cancer Research 12, 5746-5754 (2006).

12. Muller, F.L. et al. Passenger deletions generate therapeutic vulnerabilities in cancer. Nature 488, 337-+ (2012).

13. Dudani, A.K., Cummings, C., Hashemi, S. \& Ganz, P.R. ISOLATION OF A NOVEL 45-KDA PLASMINOGEN RECEPTOR FROM HUMAN ENDOTHELIAL-CELLS. Thrombosis Research 69, 185-196 (1993).

14. Nakajima, K. et al. PLASMINOGEN BINDS SPECIFICALLY TO ALPHA-ENOLASE ON RAT NEURONAL PLASMA-MEMBRANE. Journal of Neurochemistry 63, 2048-2057 (1994).

15. Johnstone, S.A., Waisman, D.M. \& Rattner, J.B. ENOLASE IS PRESENT AT THE CENTROSOME OF HELA-CELLS. Experimental Cell Research 202, 458-463 (1992).

16. Walsh, J.L., Keith, T.J. \& Knull, H.R. GLYCOLYTIC ENZYME INTERACTIONS WITH TUBULIN AND MICROTUBULES. Biochimica Et Biophysica Acta 999, 64-70 (1989).

17. Castello, A. et al. Insights into RNA Biology from an Atlas of Mammalian mRNA-Binding 
Proteins. Cell 149, 1393-1406 (2012).

18. Carpousis, A.J. The RNA Degradosome ofEscherichia coli: An mRNA-Degrading Machine Assembled on RNase E. Annual Review of Microbiology 61, 71-87 (2007).

19. Masai, H., Matsumoto, S., You, Z., Yoshizawa-Sugata, N. \& Oda, M. Eukaryotic Chromosome DNA Replication: Where, When and How?, in Annual Review of Biochemistry, Vol 79, Vol. 79. (eds. R.D. Kornberg, C.R.H. Raetz, J.E. Rothman \& J.W. Thorner) 89-130 (2010).

20. Hentze, M.W., Muckenthaler, M.U. \& Andrews, N.C. Balancing acts: Molecular control of mammalian iron metabolism. Cell 117, 285-297 (2004).

21. Costa da Silva, M. et al. Iron Induces Anti-tumor Activity in Tumor-Associated Macrophages. Frontiers in immunology 8, 1479-1479 (2017).

22. Hassannia, B., Vandenabeele, P. \& Vanden Berghe, T. Targeting Ferroptosis to Iron Out Cancer. Cancer Cell 35, 830-849 (2019).

23. Stockwell, B.R. et al. Ferroptosis: A Regulated Cell Death Nexus Linking Metabolism, Redox Biology, and Disease. Cell 171, 273-285 (2017).

24. Torti, S.V. \& Torti, F.M. Iron and cancer: more ore to be mined. Nature Reviews Cancer 13, $342-355$ (2013)

25. Zhou, B. et al. Tom20 senses iron-activated ROS signaling to promote melanoma cell pyroptosis. Cell Research 28, 1171-1185 (2018).

26. Dixon, S.J. et al. Ferroptosis: An Iron-Dependent Form of Nonapoptotic Cell Death. Cell 149, 1060-1072 (2012).

27. Chen, L., Hambright, W.S., Na, R. \& Ran, Q. Ablation of the Ferroptosis Inhibitor Glutathione Peroxidase 4 in Neurons Results in Rapid Motor Neuron Degeneration and Paralysis. Journal of Biological Chemistry 290, 28097-28106 (2015).

28. Chu, B. et al. ALOX12 is required for p53-mediated tumour suppression through a distinct ferroptosis pathway. Nature Cell Biology 21, 579-+ (2019).

29. Do Van, B. et al. Ferroptosis, a newly characterized form of cell death in Parkinson's disease that is regulated by PKC. Neurobiology of Disease 94, 169-178 (2016)

30. Jiang, L. et al. Ferroptosis as a p53-mediated activity during tumour suppression. Nature 520, 57-+ (2015). 
31. Zhang, Y. et al. BAP1 links metabolic regulation of ferroptosis to tumour suppression. Nature Cell Biology 20, 1181-+ (2018).

32. Hudson, W.H. \& Ortlund, E.A. The structure, function and evolution of proteins that bind DNA and RNA. Nature Reviews Molecular Cell Biology 15, 749-760 (2014).

33. Wang, W.Y. et al. Identification of alpha-enolase as a nuclear DNA-binding protein in the zona fasciculata but not the zona reticularis of the human adrenal cortex. Journal of Endocrinology 184, 85-94 (2005).

34. Kang, H.J., Jung, S.K., Kim, S.J. \& Chung, S.J. Structure of human alpha-enolase (hENO1), a multifunctional glycolytic enzyme. Acta crystallographica. Section D, Biological crystallography 64, 651-657 (2008).

35. Feo, S., Arcuri, D., Piddini, E., Passantino, R. \& Giallongo, A. ENO1 gene product binds to the c-myc promoter and acts as a transcriptional repressor: relationship with Myc promoter-binding protein 1 (MBP-1). Febs Letters 473, 47-52 (2000).

36. Sharova, L.V. et al. Database for mRNA Half-Life of 19977 Genes Obtained by DNA Microarray Analysis of Pluripotent and Differentiating Mouse Embryonic Stem Cells. DNA Research 16, 45-58 (2009).

37. Lau, N.-C. et al. Human Ccr4-Not complexes contain variable deadenylase subunits. Biochemical Journal 422, 443-453 (2009).

38. Chen, G., Fillebeen, C., Wang, J. \& Pantopoulos, K. Overexpression of iron regulatory protein 1 suppresses growth of tumor xenografts. Carcinogenesis 28, 785-791 (2007).

39. Chapoutot, C. et al. Liver iron excess in patients with hepatocellular carcinoma developed on viral C cirrhosis. Gut 46, 711-714 (2000).

40. Sorrentino, P. et al. Liver iron excess in patients with hepatocellular carcinoma developed on non-alcoholic steato-hepatitis. Journal of Hepatology 50, 351-357 (2009).

41. Galy, B. et al. Iron Regulatory Proteins Secure Mitochondrial Iron Sufficiency and Function. Cell Metabolism 12, 194-201 (2010).

42. Martelli, A. et al. Iron Regulatory Protein 1 Sustains Mitochondrial Iron Loading and Function in Frataxin Deficiency. Cell Metabolism 21, 311-322 (2015).

43. Shaw, G.C. et al. Mitoferrin is essential for erythroid iron assimilation. Nature 440, 96-100 (2006). 
44. Shen, S. et al. A miR-130a-YAP positive feedback loop promotes organ size and tumorigenesis. Cell Research 25, 997-1012 (2015).

45. Troadec, M.-B. et al. Targeted deletion of the mouse Mitoferrin1 gene: from anemia to protoporphyria. Blood 117, 5494-5502 (2011).

46. Sabharwal, S.S. \& Schumacker, P.T. Mitochondrial ROS in cancer: initiators, amplifiers or an Achilles' heel? Nature Reviews Cancer 14, $709-721$ (2014).

47. Badgley, M.A. et al. Cysteine depletion induces pancreatic tumor ferroptosis in mice. Science 368, 85-+ (2020).

48. Bersuker, K. et al. The CoQ oxidoreductase FSP1 acts parallel to GPX4 to inhibit ferroptosis. Nature 575, 688-+ (2019).

49. Bock, F.J. \& Tait, S.W.G. Mitochondria as multifaceted regulators of cell death. Nature Reviews Molecular Cell Biology 21, 85-100 (2020).

50. Doll, S. et al. FSP1 is a glutathione-independent ferroptosis suppressor. Nature 575, 693-+ (2019).

51. Gao, M. et al. Role of Mitochondria in Ferroptosis. Molecular Cell 73, 354-+ (2019).

52. Cloonan, S.M. et al. Mitochondrial iron chelation ameliorates cigarette smoke-induced bronchitis and emphysema in mice. Nature Medicine 22, 163-174 (2016).

53. Koppenol, W.H., Bounds, P.L. \& Dang, C.V. Otto Warburg's contributions to current concepts of cancer metabolism. Nature Reviews Cancer 11, 325-337 (2011).

54. Entelis, N. et al. A glycolytic enzyme, enolase, is recruited as a cofactor of tRNA targeting toward mitochondria in Saccharomyces cerevisiae. Genes \& Development 20, 1609-1620 (2006).

55. Hernandez-Perez, L. et al. alpha-Enolase binds to RNA. Biochimie 93, 1520-1528 (2011).

56. Rennekamp, A.J. The Ferrous Awakens. Cell 171, 1226-1227 (2017).

57. Angeli, J.P.F., Shah, R., Pratt, D.A. \& Conrad, M. Ferroptosis Inhibition: Mechanisms and Opportunities. Trends in Pharmacological Sciences 38, 489-498 (2017).

58. Reed, John C. \& Pellecchia, M. Ironing Out Cell Death Mechanisms. Cell 149, 963-965 (2012).

59. Gao, M. et al. Ferroptosis is an autophagic cell death process. Cell Research 26, 1021-1032 (2016). 
60. Hassannia, B. et al. Nano-targeted induction of dual ferroptotic mechanisms eradicates high-risk neuroblastoma. Journal of Clinical Investigation 128, 3341-3355 (2018).

61. Li, Q. et al. Inhibition of neuronal ferroptosis protects hemorrhagic brain. Jci Insight 2 (2017).

62. Wang, H. et al. Characterization of Ferroptosis in Murine Models of Hemochromatosis. Hepatology 66, 449-465 (2017).

63. Chatterjee, A. et al. MOF Acetyl Transferase Regulates Transcription and Respiration in Mitochondria. Cell 167, 722-+ (2016).

64. Salles, F.J. \& Strickland, S. Analysis of poly(A) tail lengths by PCR: the PAT assay. Methods in molecular biology (Clifton, N.J.) 118, 441-448 (1999).

65. Guan, K.-L., Yu, W., Lin, Y., Xiong, Y. \& Zhao, S. Generation of acetyllysine antibodies and affinity enrichment of acetylated peptides. Nature Protocols 5, 1583-1595 (2010).

66. Rebouche, C.J., Wilcox, C.L. \& Widness, J.A. Microanalysis of non-heme iron in animal tissues. Journal of Biochemical and Biophysical Methods 58, 239-251 (2004).

67. Birch-Machin, M.A. \& Turnbull, D.M. Assaying mitochondrial respiratory complex activity in mitochondria isolated from human cells and tissues, in Methods in Cell Biology, Vol 65: Mitochondria, Vol. 65. (eds. L.A. Pon \& E.A. Schon) 97-117 (2001).

68. Chan, A.C.Y. et al. Evaluation of the seventh edition of the American Joint Committee on Cancer tumournodemetastasis (TNM) staging system for patients undergoing curative resection of hepatocellular carcinoma: implications for the development of a refined staging system. Hpb 15, 439-448 (2013). 


\section{Figure legends}

Fig. 1 ENO1 degrades IRPI mRNA as an RNA-binding protein. a, Scatter plot showing all expression changes of genes in PLC cells expressing NTC or shENO1. Blue dots indicate downregulated genes whereas red dots indicate upregulated genes by knockdown of ENO1 (fold change $>1.5$ or $<0.67)$. b, Venn diagram of RNA-Seq and CLIP-Seq showing that the mRNA of 17 genes was bound and downregulated by ENO1. c, The mRNA levels of 17 screened genes were determined by qPCR in PLC cells expressing NTC or shENO1. d, The IRP1 mRNA levels were measured by qPCR in 17 pairs of clinically matched tumor adjacent noncancerous liver tissues (normal) and human HCC tissues (tumor). e, RNA immunoprecipitation analysis of the binding of endogenous IRPI and ENOI mRNAs by ENO1 in PLC cells overexpressing ENO1. 18s served as the negative control. f, qPCR and western blot analysis of IRP1 expression in PLC cells with ENO1 overexpression or knockdown. g, IRPI mRNA stability was determined in ENO1-overexpressing PLC cells treated with the transcriptional inhibitor actinomycin $\mathrm{D}(5 \mu \mathrm{M})$ for 0,3 or 6 hours. h, Pulse-chase analysis of IRPI mRNA stability in PLC cells with ENO1 overexpression. i, qPCR and western blot analysis of IRP1 expression in PLC cells overexpressing wild-type ENO1 or $\mathrm{ENO}^{\Delta \alpha 3 \alpha 4}, \mathrm{ENO}^{\Delta \beta 4 \beta 5}, \mathrm{ENO}^{\Delta \alpha 5 \alpha 6}$, and ENO1 ${ }^{\Delta \text { all }}$ mutants. The diagram of ENO1 protein showed its N-terminus, DNA-binding domain (DBD, $\left.\alpha_{3} \alpha_{4} \beta_{4} \beta_{5} \alpha_{5} \alpha_{6}\right)$ and C-terminus. The ENO1 ${ }^{\Delta \alpha 3 \alpha 4}$, $\mathrm{ENO}^{\Delta \beta 4 \beta 5}, \mathrm{ENO} 1^{\Delta \alpha 5 \alpha 6}$, and ENO1 ${ }^{\Delta \text { all }}$ mutants have deletions in the $\alpha_{3} \alpha_{4}$ domain, $\beta_{4} \beta_{5}$ domain, $\alpha_{5} \alpha_{6}$ domain, and $\alpha_{3} \alpha_{4} \beta_{4} \beta_{5} \alpha_{5} \alpha_{6}$ domain, respectively. $\mathbf{j}$, RNA immunoprecipitation analysis of the binding of endogenous IRP1 mRNA transcripts expressing Flag-tagged wild-type ENO1, ENO1 ${ }^{\Delta \alpha 3 \alpha 4}$, $\mathrm{ENO}^{\triangle \beta 4 \beta 5}, \mathrm{ENO}^{\Delta \alpha 5 \alpha 6}, \mathrm{ENO}^{\triangle \mathrm{all}}, \mathrm{ENO}^{\mathrm{S} 40 \mathrm{~A}}$, or ENO1 ${ }^{\mathrm{D} 245 \mathrm{R}}$ in PLC cells. ENO1 ${ }^{\mathrm{S} 40 \mathrm{~A}}$ and $\mathrm{ENO1}{ }^{\mathrm{D} 245 \mathrm{R}}$ are two catalytically dead mutants of ENO1. k, Western blot analysis of IRP1 protein in PLC cells overexpressing ENO1, MBP1, or ENO1 ${ }^{\mathrm{DBD}}$ mutant. l, m, Flag-tagged EV or ENO1 plasmids were co-transfected with pGL3-EV, pGL3-IRP1-5'UTR and pGL3-IRP1-3'UTR plasmids (l) or pGL3-EV, pGL3-IRP1-5'UTR and pGL3-IRP1-CpG-rich deletion plasmids (m) into HEK293 cells followed by dual-luciferase analysis. n, The working model depicts that ENO1 degrades IRPI mRNA as an RNA-binding protein. In brief, ENO1 binds to the CpG-rich region of IRP1 5'UTR via its DNA-binding domain and ultimately promotes mRNA degradation of IRP1. Data are presented as the mean \pm s.d. of three independent experiments $(\mathbf{c}, \mathbf{e}-\mathbf{j}, \mathbf{l}, \mathbf{m})$ or mean \pm s.e.m. $(\mathbf{d}) .{ }^{*} P<0.05$ 
compared with the indicated groups.

Fig. 2 ENO1 recruits CNOT6 to degrade IRP1 mRNA. a, Analysis of IRPI poly (A) tail length in PLC cells expressing Flag-tagged ENO1 using semi-qPCR. GAPDH served as negative control. b, HEK293T cells were transfected with HA-ENO1 plasmids together with Flag-EV or Flag-tagged plasmids expressing CNOT3, CNOT6, CNOT7, CNOT9 or CNOT10. c, GST pull-down of His-CNOT6 by GST-ENO1 using proteins purified in E. coli, followed by western blot analysis with anti-CNOT6 and anti-GST antibodies. d, HEK293T cells were transfected with HA-CNOT6 plasmids together with Flag-EV or Flag-ENO1 plasmids. Cell lysates were immunoprecipitated with anti-Flag antibody or IgG, followed by western blot analysis. e, qPCR and western blot analysis of the expression of IRP1 in PLC cells expressing Flag-tagged CNOT6. f, HEK293T cells were transfected with HA-ENO1 plasmids together with Flag-EV or Flag-tagged plasmids expressing CNOT6, CNOT6-LR or CNOT6-NU. g, HEK293T cells were transfected with HA-CNOT6 plasmids together with Flag-EV or Flag-tagged plasmids expressing wild-type ENO1, ENO1-N terminus or ENO1-DBD+C terminus. h, HEK293T cells were transfected with HA-CNOT6 plasmids together with Flag-EV or Flag-tagged plasmids expressing the indicated domains of ENO1. i, HEK293T cells were transfected with HA-CNOT6-LR domain plasmids together with Flag-EV or Flag-ENO1-C terminus plasmids. j, PLC cells stably overexpressing CNOT6 were further infected with viruses expressing NTC and shENO1. The protein levels of IRP1 were measured by western blots. k, The working model depicts that ENO1 degrades IRPI mRNA by recruiting CNOT6. In brief, ENO1 binds to IRPI mRNA via its DNA-binding domain and interacts with the leucine repeat (LR) domain of CNOT6 via its C terminus, whereby the nuclease (NU) domain of CNOT6 promoted IRPI mRNA degradation by deadenylating the IRPI 3'UTR. Data are presented as the mean \pm s.d. of three independent experiments (e). $* P<0.05$ compared with the indicated groups.

Fig. 3 ENO1 promotes liver cancer by inhibiting the IRP1/Mfrn1 axis. a, Cell growth curves were measured in PLC cells overexpressing IRP1 in the absence or presence of $200 \mu \mathrm{M}$ ferrous iron. b, PLC cells stably expressing EV or IRP1 were injected subcutaneously into nude mice ( $\mathrm{n}=5$ for each group). Tumor sizes were measured starting at 10 days after inoculation. c, Serum iron was 
measured by an automatic biochemical analyzer from 55 healthy human samples and 143 human HCC samples. d, Heat map analysis of qPCR data showing the mRNA expression of mitochondrial iron-sulfur cluster synthesis-related genes when IRP1 was knocked down in PLC cells. Blue bars indicate downregulated genes whereas red bars indicate upregulated genes. e, qPCR and western blot analysis of the expression of Mfrn1 in PLC cells with IRP1 overexpression in the presence of ferrous iron $(200 \mu \mathrm{M})$. f, Cell growth curves were determined in PLC cells overexpressing Mfrn1 in the absence or presence of $200 \mu \mathrm{M}$ ferrous iron. g, qPCR and western blot analysis of the expression of IRP1 and Mfrn1 in PLC cells overexpressing ENO1 in the presence of ferrous iron. $\mathbf{h}$, PLC cells overexpressing ENO1 were further infected with viruses overexpressing IRP1 in the presence of ferrous iron. Protein levels of Mfrn1 were measured by western blots. $\mathbf{i}, \mathbf{j}$, PLC cells overexpressing ENO1 were further infected with viruses overexpressing IRP1 or Mfrn1 in the presence of ferrous iron (i). PLC cells with IRP1 knockdown were further infected with viruses overexpressing Mfrn1 in the presence of ferrous iron (j). Equal numbers of PLC cells were injected subcutaneously into the flanks of nude mice ( $n=5$ for each group). Tumor sizes were measured starting at 10 days after inoculation. k, l, Plasmids expressing human YAP-5SA alone or human YAP-5SA plus mouse-IRP1 or mouse-Mfrn1 together with plasmids expressing PB transposase were delivered into mice by hydrodynamic injection ( $\mathrm{n}=5$ in each group) (k). Plasmids expressing human YAP-5SA together with plasmids expressing PB transposase were delivered by hydrodynamic injection into wide type or $M f r n I^{+/-}$mice ( $\mathrm{n}=5$ in each group) (l). YAP-5SA induced liver tumorigenesis was analyzed approximately 100 days after injection. RFP served as a control. Liver/body weight ratios were measured at the end of the experiment. Data are presented as the mean \pm s.d. of three independent experiments (a, e-g) or mean \pm s.e.m. $(\mathbf{b}, \mathbf{c}, \mathbf{i}-\mathbf{l}) . * P<0.05$ compared with the indicated groups.

\section{Fig. 4 The ENO1/IRP1/Mfrn1 axis regulates ferroptosis by modulating mitochondrial iron} homeostasis. a, Mitochondrial iron was measured in PLC cells overexpressing ENO1, IRP1 or Mfrn1 in the presence of ferrous iron. b, PLC cells overexpressing IRP1 were further infected with viruses expressing NTC or shMfrn1 in the presence of ferrous iron, followed by mitochondrial iron detection using a microplate reader. c, PLC cells overexpressing ENO1 were further infected with viruses overexpressing IRP1 or Mfrn1 in the presence of ferrous iron, followed by mitochondrial 
iron detection using a microplate reader. d, Cell death was measured by flow cytometry using propidium iodide (PI) staining in PLC cells overexpressing IRP1 or Mfrn1 in the absence or presence of ferrous iron. e, f, Bar graphs showing the cell death ratio by flow cytometry analysis in PLC cells overexpressing IRP1 (e) or Mfrn1 (f) treated with $200 \mu \mathrm{M}$ ferrous iron combined with 2 $\mu \mathrm{M}$ Ferr-1, $5 \mu \mathrm{M}$ deferiprone, $5 \mu \mathrm{M}$ Z-VAD-FMK, $2 \mu \mathrm{M}$ Nec-1, or $2 \mathrm{mM}$ 3-MA, respectively. g, Lipid peroxidation was assessed by flow cytometry after C11-BODIPY staining in PLC cells overexpressing IRP1 or Mfrn1 in the absence or presence of ferrous iron. $\mathbf{h}$, Transmission electron microscopy analysis of PLC cells overexpressing IRP1 or Mfrn1 treated with ferrous iron. i, j, PLC cells overexpressing IRP1 were further infected with viruses expressing NTC or shMfrn1 in the absence or presence of ferrous iron. Cell death (i) and lipid peroxidation (j) were measured by flow cytometry using PI staining and C11-BODIPY staining, respectively. k, PLC cells with ENO1 knockdown were further infected with viruses expressing NTC or shRNAs targeting IRP1 or Mfrn1 in the presence of ferrous iron. The cell death ratio was measured by flow cytometry using PI staining. l, m, PLC cells with ENO1 knockdown were further infected with viruses expressing NTC or shRNAs targeting IRP1 (l) or Mfrn1 (m) in the presence of ferrous iron. Lipid peroxidation was assessed by flow cytometry after C11-BODIPY staining. Data are presented as the mean \pm s.d. of three independent experiments (a-f, i, k). $* P<0.05$ compared with the indicated groups.

Fig. 5 IRP1 and Mfrn1 deficiency predicts poor clinical prognosis. a, ENO1, IRP1 and Mfrn1 protein levels were measured by western blots using the paired adjacent noncancerous liver tissues (N) and human HCC tissues (T). Ponceau staining served as a loading control. b, ENO1, IRP1 and Mfrn1 protein levels were measured by western blots using the paired adjacent noncancerous liver tissues $(\mathrm{N})$ and cancerous liver tissues (T) in YAP-5SA-induced mouse HCC. Ponceau staining served as a loading control. c, Representative IHC images of ENO1, IRP1 and Mfrn1 staining were shown in normal liver tissues (normal) and HCC specimens of different clinical stages (I-IV). d, e, Statistical quantification of the mean optical density (MOD) values of IRP1 (d) and Mfrn1 (e) staining in IHC assays between normal liver tissues and HCC specimens with different clinical stages (I-IV). f, g, Kaplan-Meier curves with univariate analyses of patients with low versus high IRP1 (f) or Mfrn1 (g) expression. h, Representative IHC images of ENO1, IRP1 and Mfrn1 staining in serial sections were shown. Data are presented as the mean \pm s.e.m. (d, e). ${ }^{*} P<0.05$ compared 
with the normal groups.

Fig. 6 A schematic model describing the ENO1/IRP1/Mfrn1 axis in regulating ferroptosis. A schematic model shows that ENO1, as an RNA-binding protein, recruits CNOT6 to accelerate the mRNA degradation of iron regulatory protein 1 (IRP1) in HCC cells. Consequently, ENO1, via inhibition of IRP1/Mfrn1 axis, suppresses mitochondrial iron-induced ferroptosis. 
a

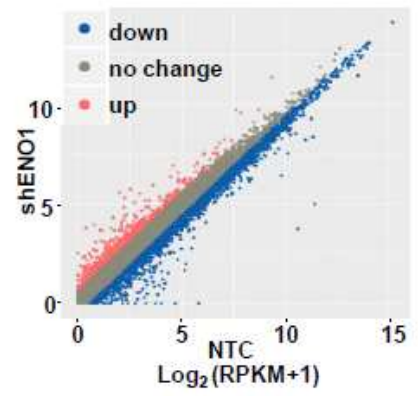

b

Downregulated ENO1 CLIP-Seq
by ENO1

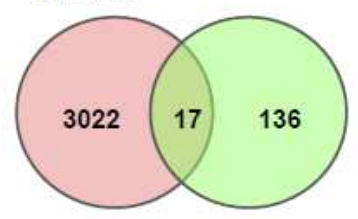

f

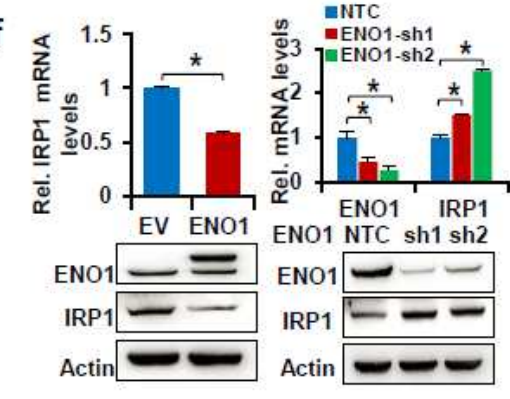

i

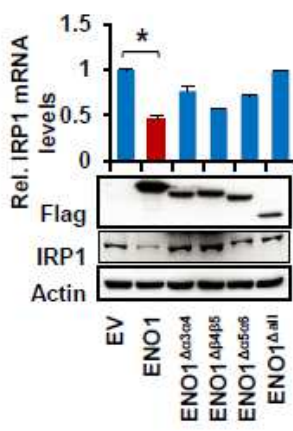

I

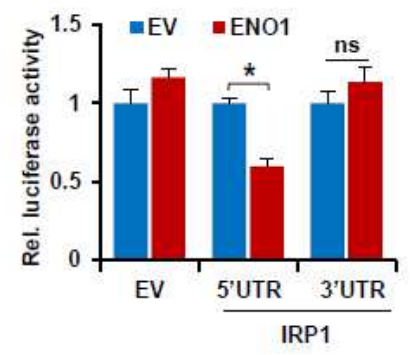

m

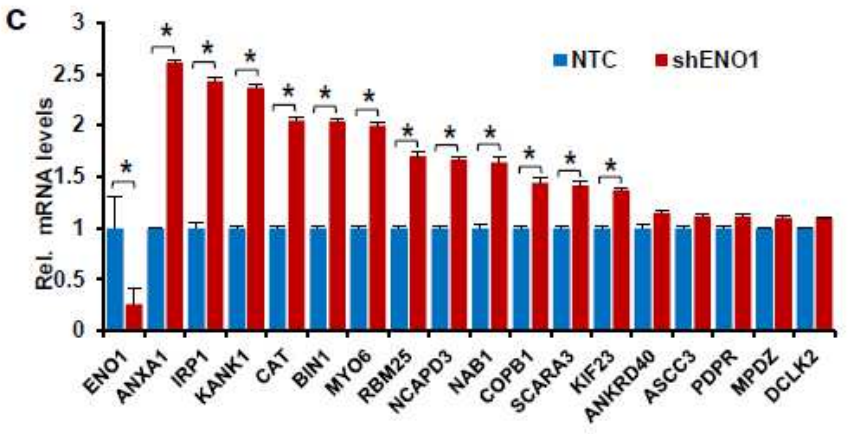

d
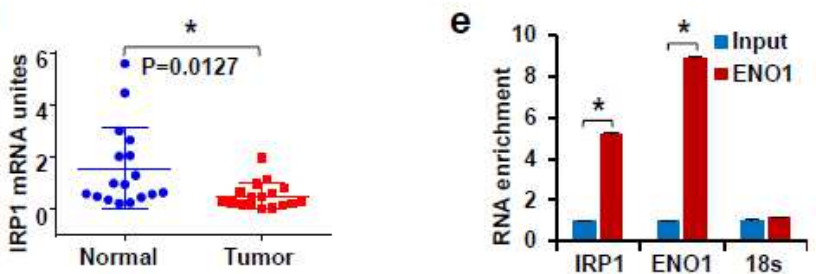

h
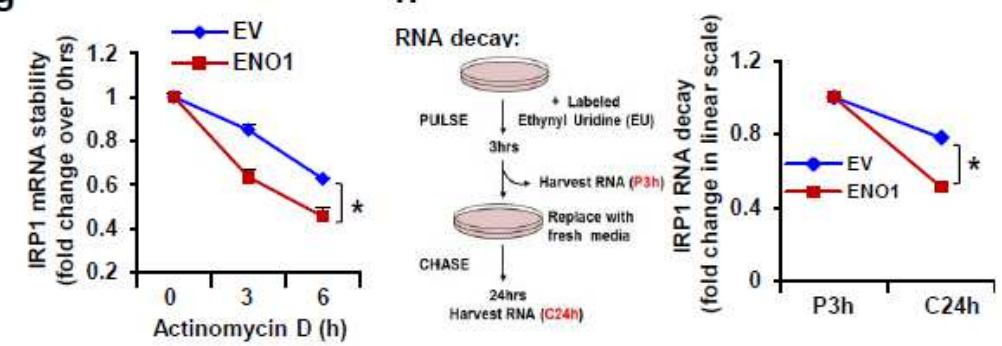

k
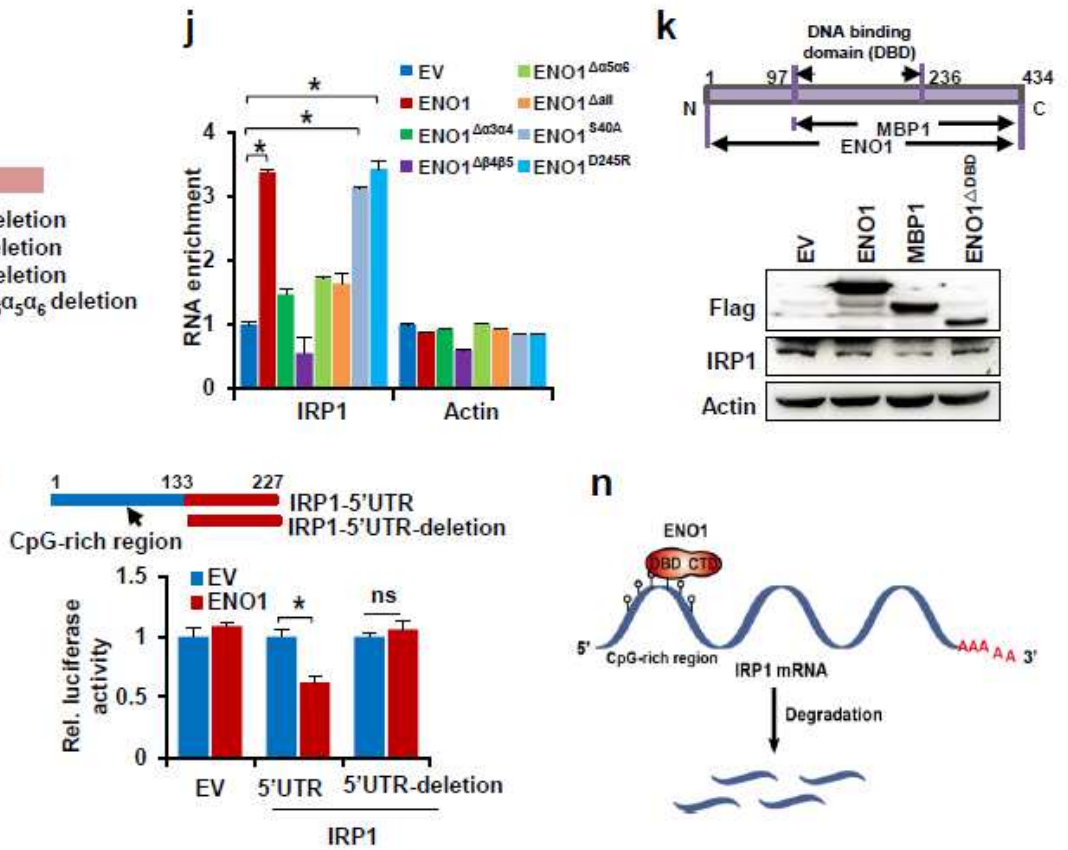

n

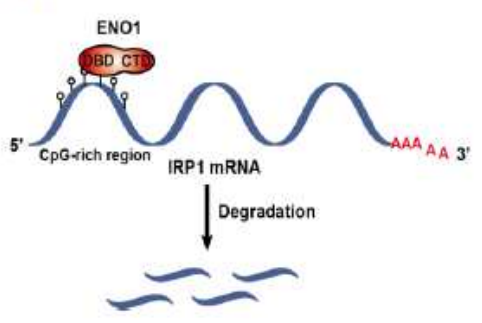

Figure 1

EN01 degrades IRP1 mRNA as an RNA-binding protein. a, Scatter plot showing all expression changes of genes in PLC cells expressing NTC or shENO1. Blue dots indicate downregulated genes whereas red dots indicate upregulated genes by knockdown of ENO1 (fold change $>1.5$ or $<0.67$ ). b, Venn diagram of RNA- 
Seq and CLIP-Seq showing that the mRNA of 17 genes was bound and downregulated by ENO1. c, The mRNA levels of 17 screened genes were determined by qPCR in PLC cells expressing NTC or shENO1. d, The IRP1 mRNA levels were measured by qPCR in 17 pairs of clinically matched tumor adjacent noncancerous liver tissues (normal) and human HCC tissues (tumor). e, RNA immunoprecipitation analysis of the binding of endogenous IRP1 and ENO1 mRNAs by ENO1 in PLC cells overexpressing EN01. 18s served as the negative control. f, qPCR and western blot analysis of IRP1 expression in PLC cells with ENO1 overexpression or knockdown. g, IRP1 mRNA stability was determined in ENO1overexpressing PLC cells treated with the transcriptional inhibitor actinomycin $D(5 \mu \mathrm{M})$ for 0,3 or 6 hours. h, Pulse-chase analysis of IRP1 mRNA stability in PLC cells with ENO1 overexpression. i, qPCR and western blot analysis of IRP1 expression in PLC cells overexpressing wild-type ENO1 or ENO1 $\triangle a 3 a 4$, ENO1 $\triangle \beta 4 \beta 5$, EN01 $\triangle a 5 a 6$, and EN01 $\Delta$ all mutants. The diagram of EN01 protein showed its $N$-terminus,

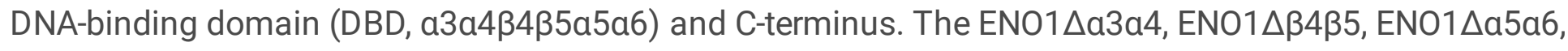
and EN01 $\triangle$ all mutants have deletions in the a3a4 domain, $\beta 4 \beta 5$ domain, a5a6 domain, and a3a4ß435a5a6 domain, respectively. j, RNA immunoprecipitation analysis of the binding of endogenous IRP1 mRNA transcripts expressing Flag-tagged wild-type ENO1, EN01 $\triangle a 3 a 4, E N 01 \Delta \beta 4 \beta 5$, ENO1 $\Delta a 5 a 6$, EN01 $\triangle$ all, EN01S40A, or EN01D245R in PLC cells. EN01S40A and ENO1D245R are two catalytically dead mutants of ENO1. $k$, Western blot analysis of IRP1 protein in PLC cells overexpressing ENO1, MBP1, or EN01هDBD mutant. I, m, Flag-tagged EV or ENO1 plasmids were co-transfected with pGL3-EV, pGL3IRP1-5'UTR and pGL3-IRP1-3'UTR plasmids (I) or pGL3-EV, pGL3-IRP1-5'UTR and pGL3-IRP1-CpG-rich deletion plasmids $(\mathrm{m})$ into HEK293 cells followed by dual-luciferase analysis. $n$, The working model depicts that ENO1 degrades IRP1 mRNA as an RNA-binding protein. In brief, ENO1 binds to the CpG-rich region of IRP1 5'UTR via its DNA-binding domain and ultimately promotes mRNA degradation of IRP1. Data are presented as the mean \pm s.d. of three independent experiments $(c, e-j, l, m)$ or mean \pm s.e.m. (d). * $\mathrm{P}<0.05$ compared with the indicated groups. 
a

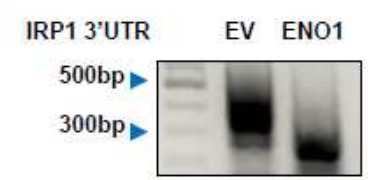

GAPDH 3'UTR

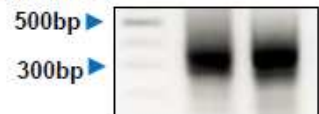

d

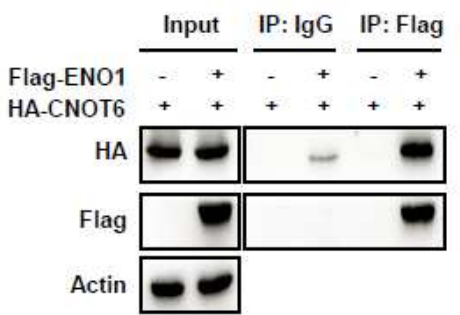

b

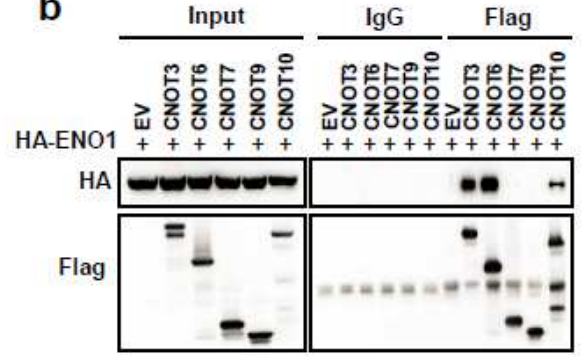

C

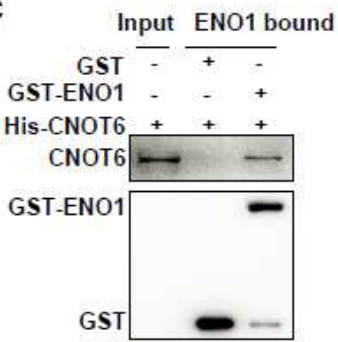

g
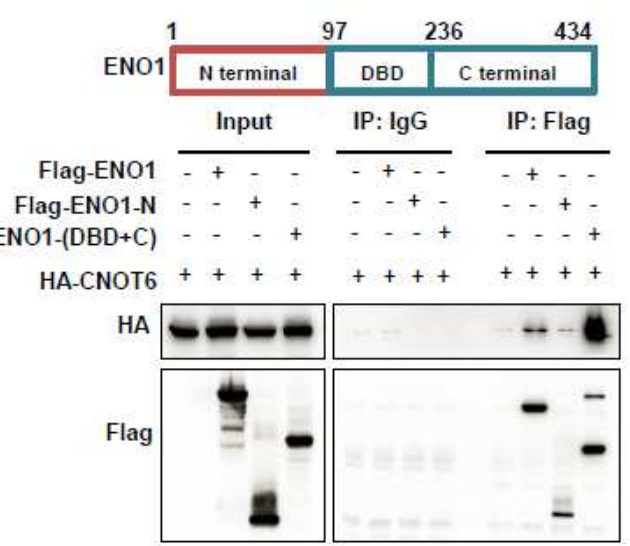

f

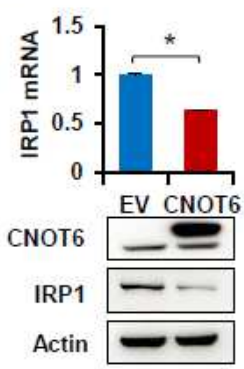

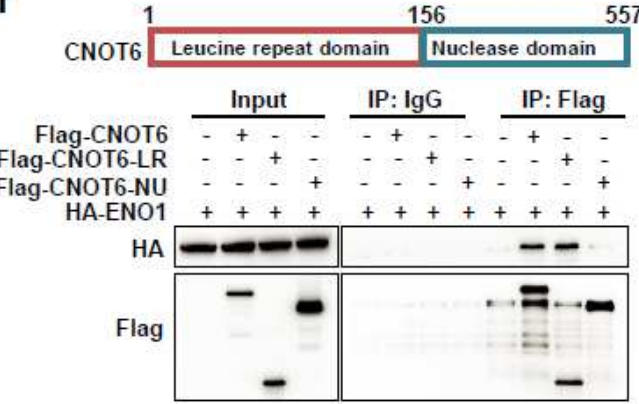

h
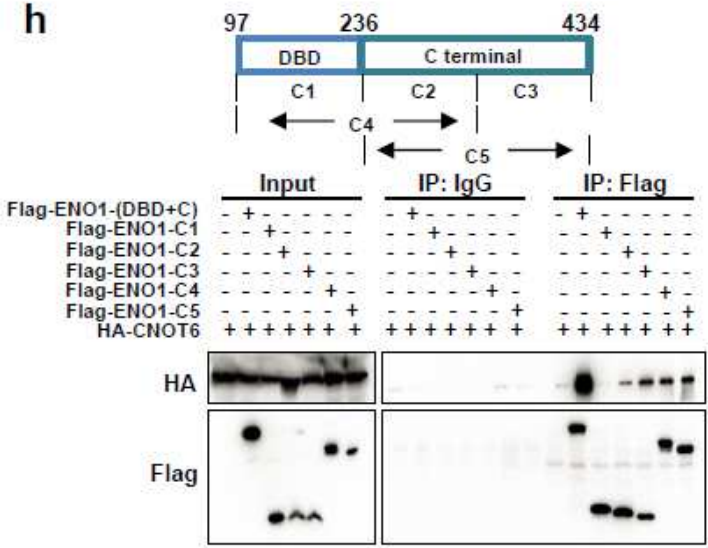

i

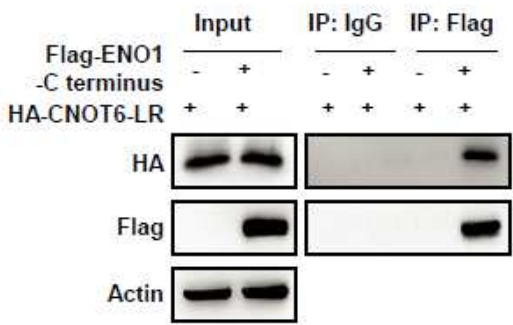

k

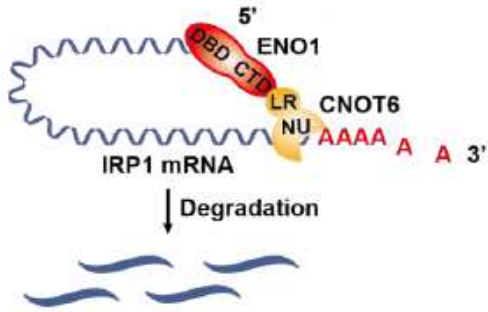

\section{Figure 2}

ENO1 recruits CNOT6 to degrade IRP1 mRNA. a, Analysis of IRP1 poly (A) tail length in PLC cells expressing Flag-tagged ENO1 using semi-qPCR. GAPDH served as negative control. b, HEK293T cells were transfected with HA-ENO1 plasmids together with Flag-EV or Flag-tagged plasmids expressing CNOT3, CNOT6, CNOT7, CNOT9 or CNOT10. c, GST pull-down of His-CNOT6 by GST-ENO1 using proteins purified in E. coli, followed by western blot analysis with anti-CNOT6 and anti-GST antibodies. d, 
HEK293T cells were transfected with HA-CNOT6 plasmids together with Flag-EV or Flag-ENO1 plasmids. Cell lysates were immunoprecipitated with anti-Flag antibody or IgG, followed by western blot analysis. e, qPCR and western blot analysis of the expression of IRP1 in PLC cells expressing Flag-tagged CNOT6. $\mathrm{f}$, HEK293T cells were transfected with HA-ENO1 plasmids together with Flag-EV or Flag-tagged plasmids expressing CNOT6, CNOT6-LR or CNOT6-NU. g, HEK293T cells were transfected with HA-CNOT6 plasmids together with Flag-EV or Flag-tagged plasmids expressing wild-type ENO1, ENO1-N terminus or ENO1DBD $+C$ terminus. h, HEK293T cells were transfected with HA-CNOT6 plasmids together with Flag-EV or Flag-tagged plasmids expressing the indicated domains of ENO1. i, HEK293T cells were transfected with HA-CNOT6-LR domain plasmids together with Flag-EV or Flag-ENO1-C terminus plasmids. j, PLC cells stably overexpressing CNOT6 were further infected with viruses expressing NTC and shENO1. The protein levels of IRP1 were measured by western blots. $k$, The working model depicts that EN01 degrades IRP1 mRNA by recruiting CNOT6. In brief, ENO1 binds to IRP1 mRNA via its DNA-binding domain and interacts with the leucine repeat (LR) domain of CNOT6 via its $C$ terminus, whereby the nuclease (NU) domain of CNOT6 promoted IRP1 mRNA degradation by deadenylating the IRP1 3'UTR. Data are presented as the mean \pm s.d. of three independent experiments $(e) .{ }^{*}<0.05$ compared with the indicated groups. 
a

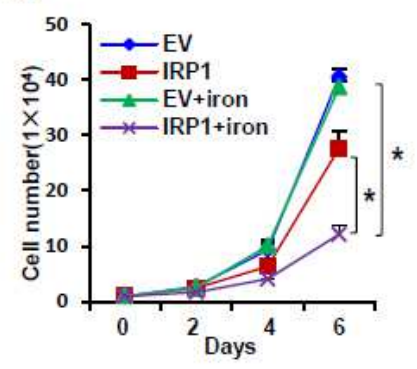

d IRP1-sh1 IRP1-sh2

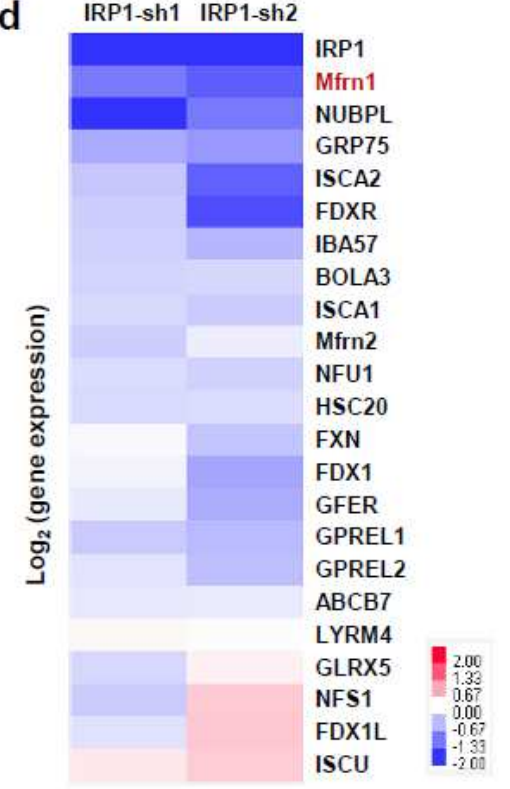

i

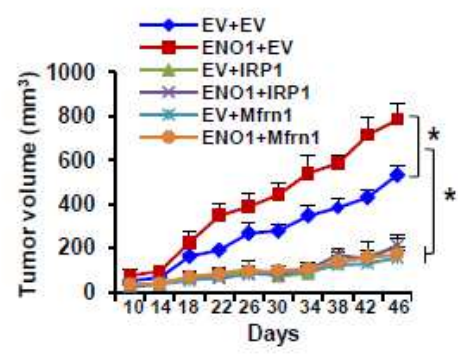

j

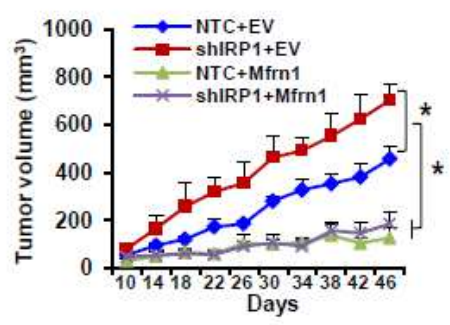

b

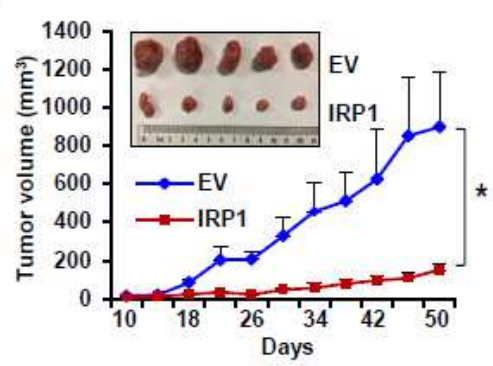

e

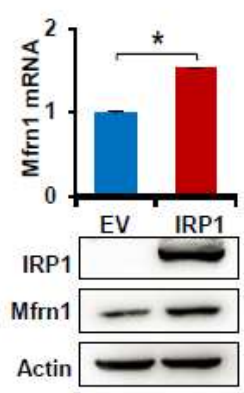

f

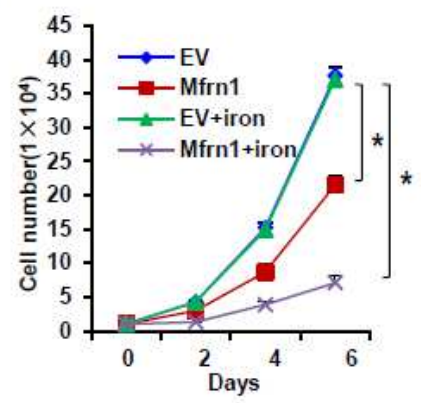

C

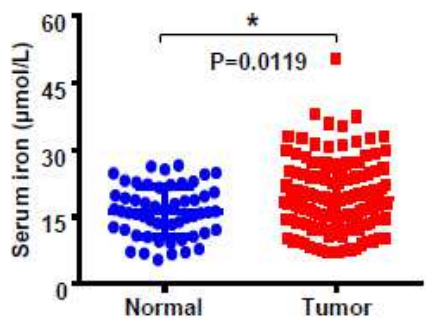

g
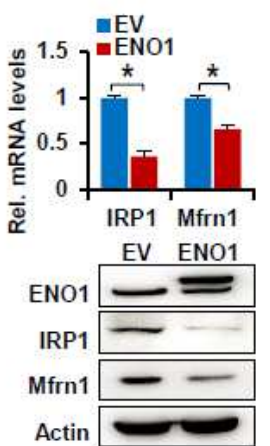

h
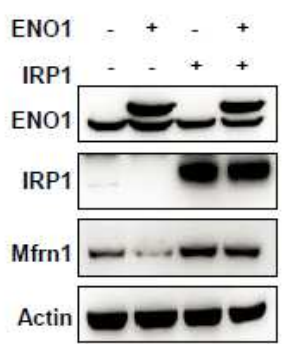

k

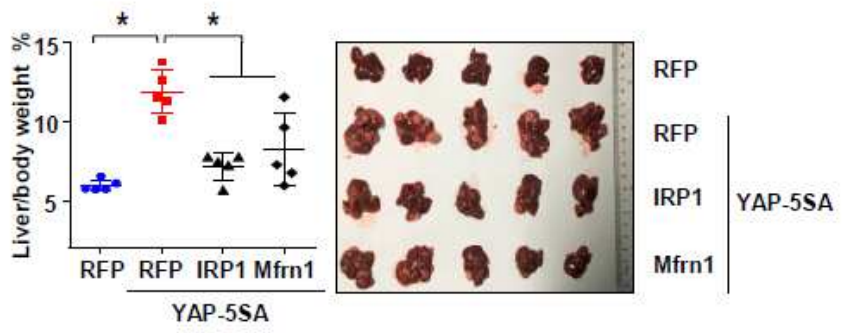

I

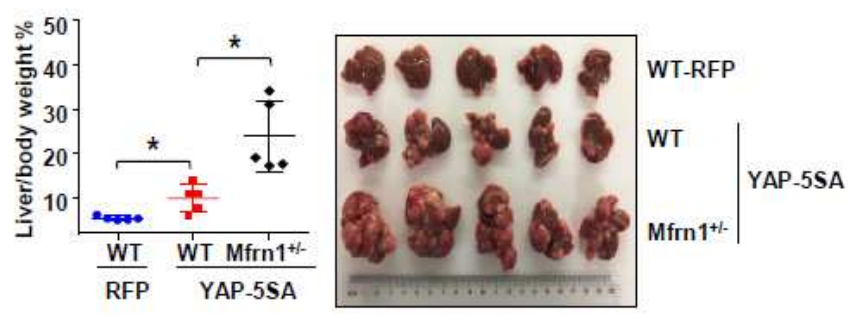

Figure 3

EN01 promotes liver cancer by inhibiting the IRP1/Mfrn1 axis. a, Cell growth curves were measured in PLC cells overexpressing IRP1 in the absence or presence of $200 \mu \mathrm{M}$ ferrous iron. b, PLC cells stably expressing EV or IRP1 were injected subcutaneously into nude mice ( $\mathrm{n}=5$ for each group). Tumor sizes were measured starting at 10 days after inoculation. c, Serum iron was measured by an automatic biochemical analyzer from 55 healthy human samples and 143 human HCC samples. d, Heat map 
analysis of qPCR data showing the mRNA expression of mitochondrial iron-sulfur cluster synthesisrelated genes when IRP1 was knocked down in PLC cells. Blue bars indicate downregulated genes whereas red bars indicate upregulated genes. e, qPCR and western blot analysis of the expression of Mfrn1 in PLC cells with IRP1 overexpression in the presence of ferrous iron $(200 \mu \mathrm{M})$. $\mathrm{f}$, Cell growth curves were determined in PLC cells overexpressing Mfrn1 in the absence or presence of $200 \mu \mathrm{M}$ ferrous iron. $\mathrm{g}$, qPCR and western blot analysis of the expression of IRP1 and Mfrn1 in PLC cells overexpressing ENO1 in the presence of ferrous iron. h, PLC cells overexpressing ENO1 were further infected with viruses overexpressing IRP1 in the presence of ferrous iron. Protein levels of Mfrn1 were measured by western blots. i, j, PLC cells overexpressing ENO1 were further infected with viruses overexpressing IRP1 or Mfrn1 in the presence of ferrous iron (i). PLC cells with IRP1 knockdown were further infected with viruses overexpressing Mfrn1 in the presence of ferrous iron (j). Equal numbers of PLC cells were injected subcutaneously into the flanks of nude mice ( $n=5$ for each group). Tumor sizes were measured starting at 10 days after inoculation. $k$, l, Plasmids expressing human YAP-5SA alone or human YAP-5SA plus mouse-IRP1 or mouse-Mfrn 1 together with plasmids expressing PB transposase were delivered into mice by hydrodynamic injection ( $n=5$ in each group) $(k)$. Plasmids expressing human YAP-5SA together with plasmids expressing PB transposase were delivered by hydrodynamic injection into wide type or Mfrn1+/mice ( $n=5$ in each group) (I). YAP-5SA induced liver tumorigenesis was analyzed approximately 100 days after injection. RFP served as a control. Liver/body weight ratios were measured at the end of the experiment. Data are presented as the mean \pm s.d. of three independent experiments $(a, e-g)$ or mean \pm s.e.m. (b, c, i-l). * $\mathrm{P}<0.05$ compared with the indicated groups. 
a

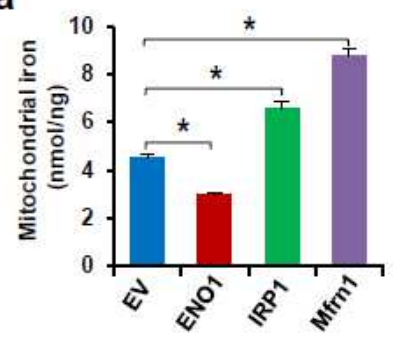

d

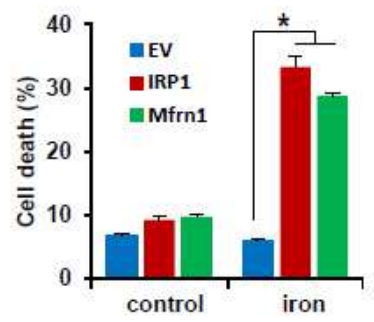

b

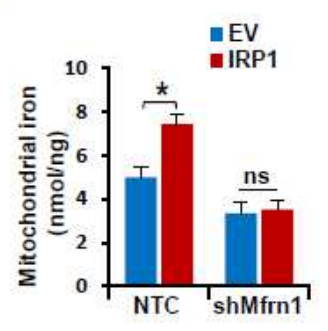

C
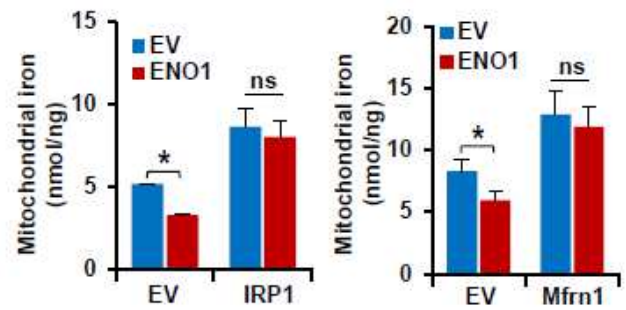

f

g

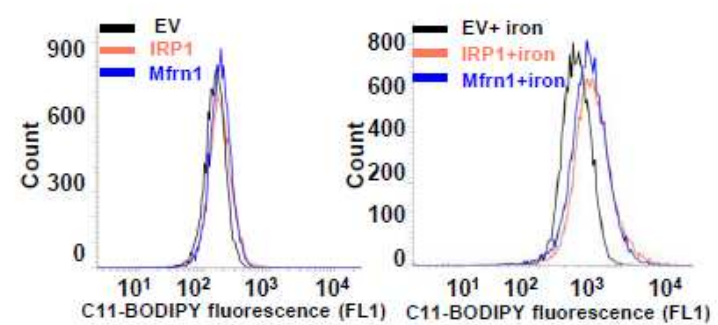

i

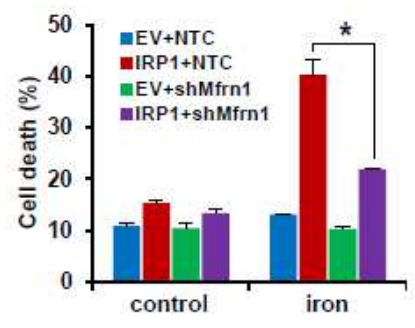

k

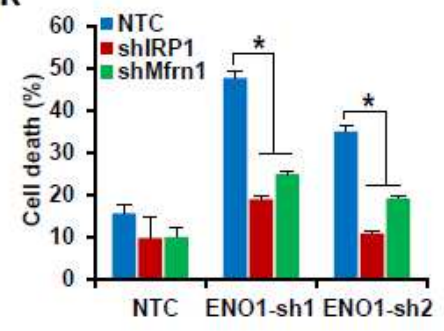

e

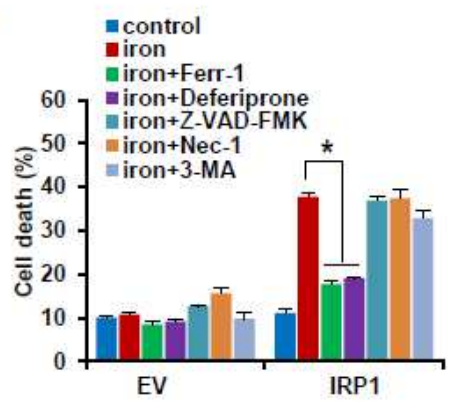

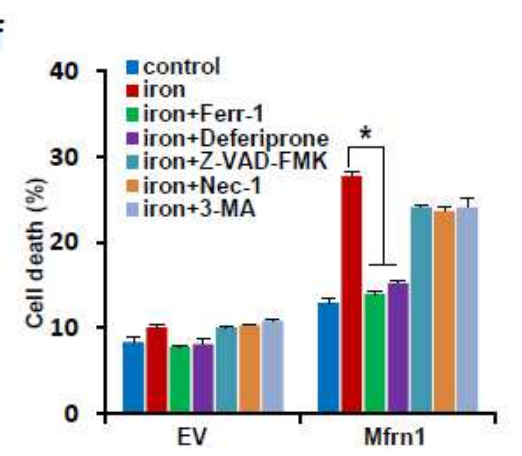
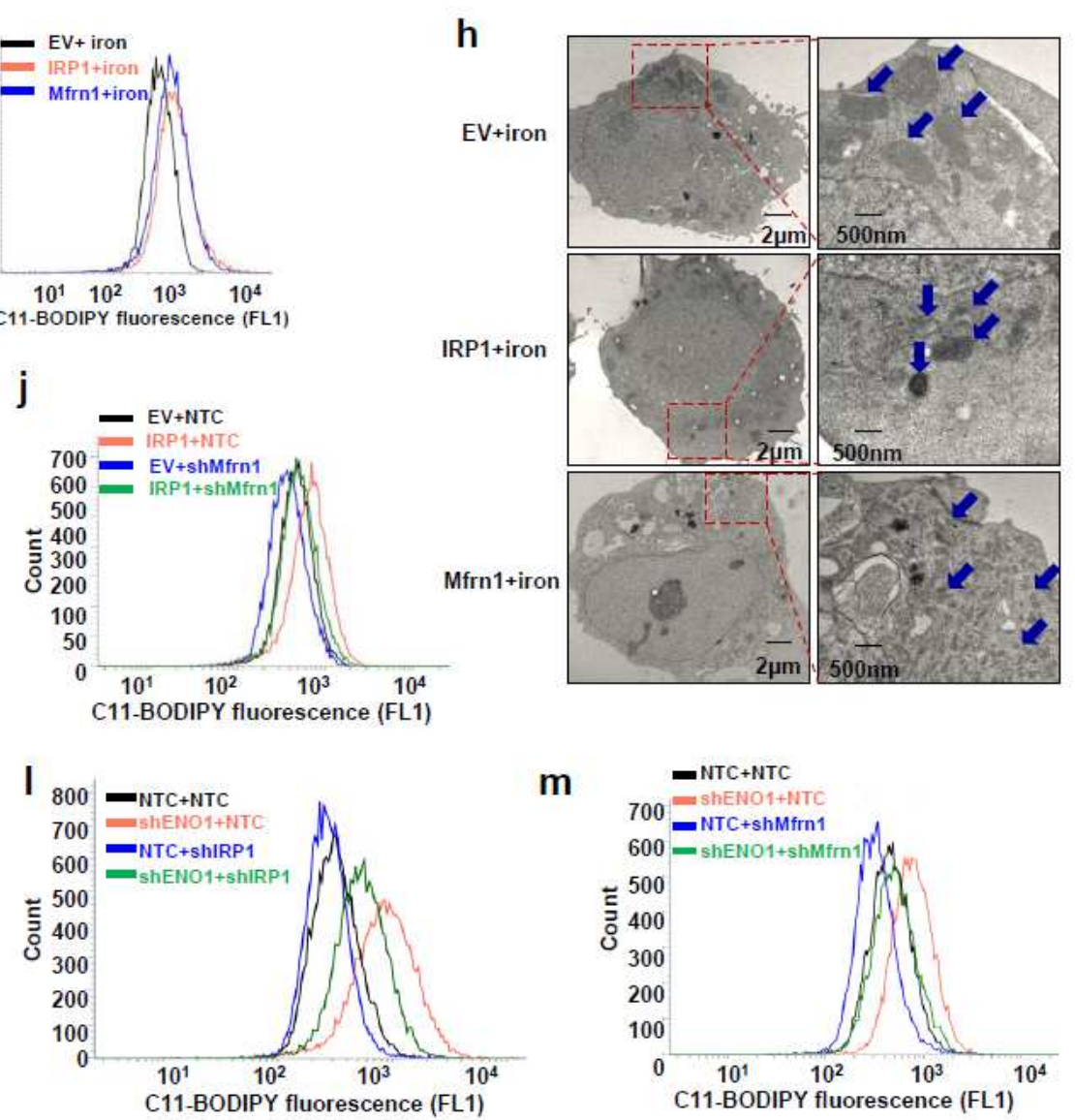

\section{Figure 4}

The EN01/IRP1/Mfrn1 axis regulates ferroptosis by modulating mitochondrial iron homeostasis. a, Mitochondrial iron was measured in PLC cells overexpressing EN01, IRP1 or Mfrn1 in the presence of ferrous iron. b, PLC cells overexpressing IRP1 were further infected with viruses expressing NTC or shMfrn1 in the presence of ferrous iron, followed by mitochondrial iron detection using a microplate reader. c, PLC cells overexpressing EN01 were further infected with viruses overexpressing IRP1 or Mfrn1 
in the presence of ferrous iron, followed by mitochondrial iron detection using a microplate reader. $d$, Cell death was measured by flow cytometry using propidium iodide (PI) staining in PLC cells overexpressing IRP1 or Mfrn 1 in the absence or presence of ferrous iron. e, $f$, Bar graphs showing the cell death ratio by flow cytometry analysis in PLC cells overexpressing IRP1 (e) or Mfrn1 (f) treated with $200 \mu \mathrm{M}$ ferrous iron combined with $2 \mu \mathrm{M}$ Ferr-1, $5 \mu \mathrm{M}$ deferiprone, $5 \mu \mathrm{M}$ Z-VAD-FMK, $2 \mu \mathrm{M}$ Nec-1, or $2 \mathrm{mM}$ 3-MA, respectively. $\mathrm{g}$, Lipid peroxidation was assessed by flow cytometry after C11-BODIPY staining in PLC cells overexpressing IRP1 or Mfrn1 in the absence or presence of ferrous iron. h, Transmission electron microscopy analysis of PLC cells overexpressing IRP1 or Mfrn1 treated with ferrous iron. i, j, PLC cells overexpressing IRP1 were further infected with viruses expressing NTC or shMfrn1 in the absence or presence of ferrous iron. Cell death (i) and lipid peroxidation (j) were measured by flow cytometry using PI staining and C11-BODIPY staining, respectively. k, PLC cells with ENO1 knockdown were further infected with viruses expressing NTC or shRNAs targeting IRP1 or Mfrn 1 in the presence of ferrous iron. The cell death ratio was measured by flow cytometry using PI staining. I, m, PLC cells with ENO1 knockdown were further infected with viruses expressing NTC or shRNAs targeting IRP1 (I) or Mfrn1 (m) in the presence of ferrous iron. Lipid peroxidation was assessed by flow cytometry after C11-BODIPY staining. Data are presented as the mean \pm s.d. of three independent experiments $(a-f, i, k) .{ }^{*} P<0.05$ compared with the indicated groups. 


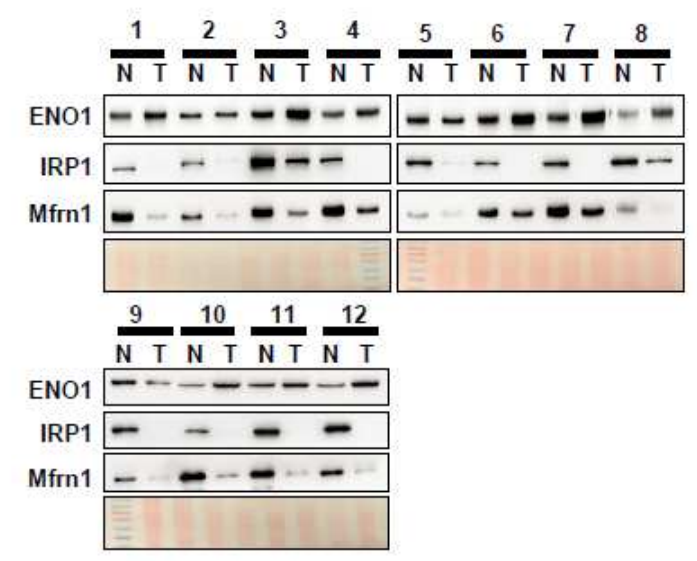

C

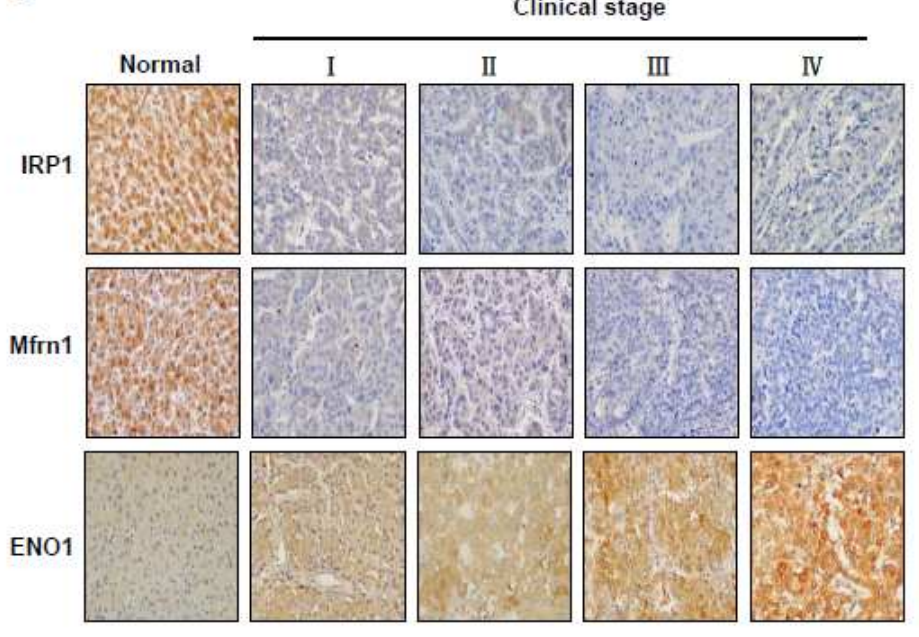

f

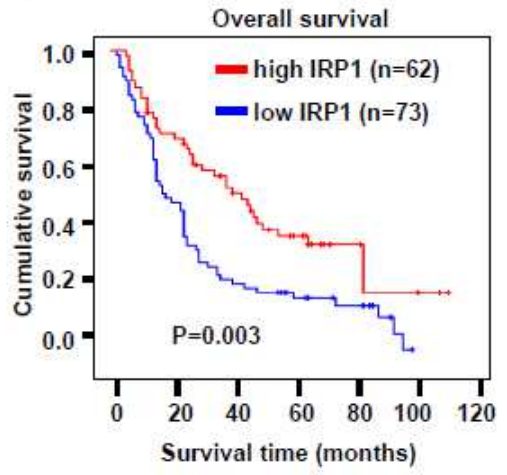

g

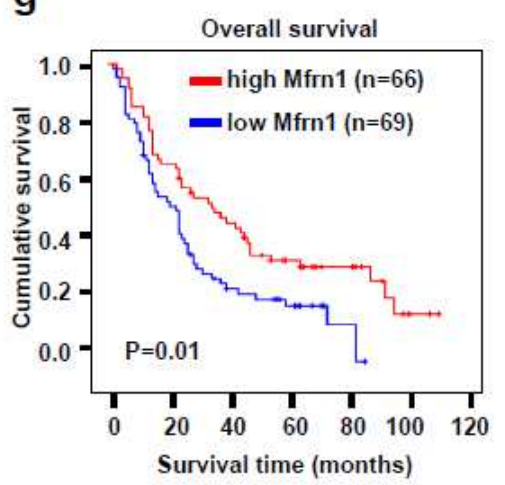

d

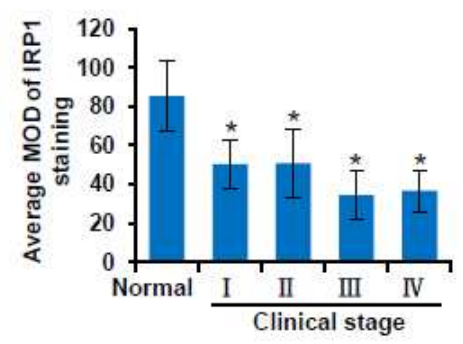

e

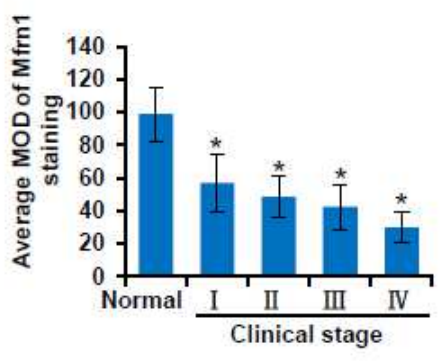

h

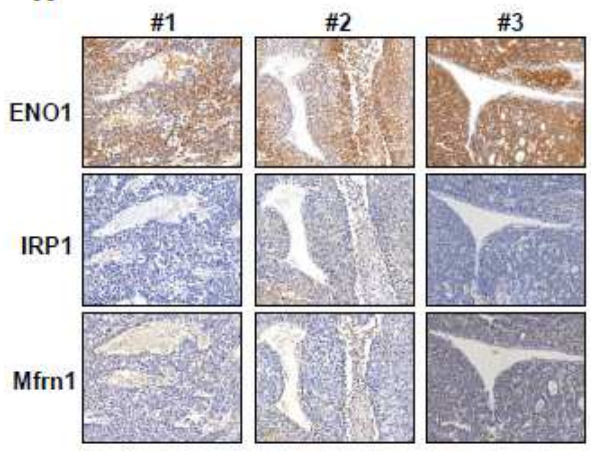

\section{Figure 5}

IRP1 and Mfrn1 deficiency predicts poor clinical prognosis. a, EN01, IRP1 and Mfrn1 protein levels were measured by western blots using the paired adjacent noncancerous liver tissues $(\mathrm{N})$ and human HCC tissues $(T)$. Ponceau staining served as a loading control. b, EN01, IRP1 and Mfrn1 protein levels were measured by western blots using the paired adjacent noncancerous liver tissues $(\mathrm{N})$ and cancerous liver tissues (T) in YAP-5SA-induced mouse HCC. Ponceau staining served as a loading control. c, 
Representative IHC images of ENO1, IRP1 and Mfrn1 staining were shown in normal liver tissues (normal) and HCC specimens of different clinical stages (I-IV). d, e, Statistical quantification of the mean optical density (MOD) values of IRP1 (d) and Mfrn1 (e) staining in IHC assays between normal liver tissues and HCC specimens with different clinical stages (I-IV). f, g, Kaplan-Meier curves with univariate analyses of patients with low versus high IRP1 (f) or Mfrn1 (g) expression. h, Representative IHC images of ENO1, IRP1 and Mfrn1 staining in serial sections were shown. Data are presented as the mean \pm s.e.m. (d, e). *P $<0.05$ compared with the normal groups.

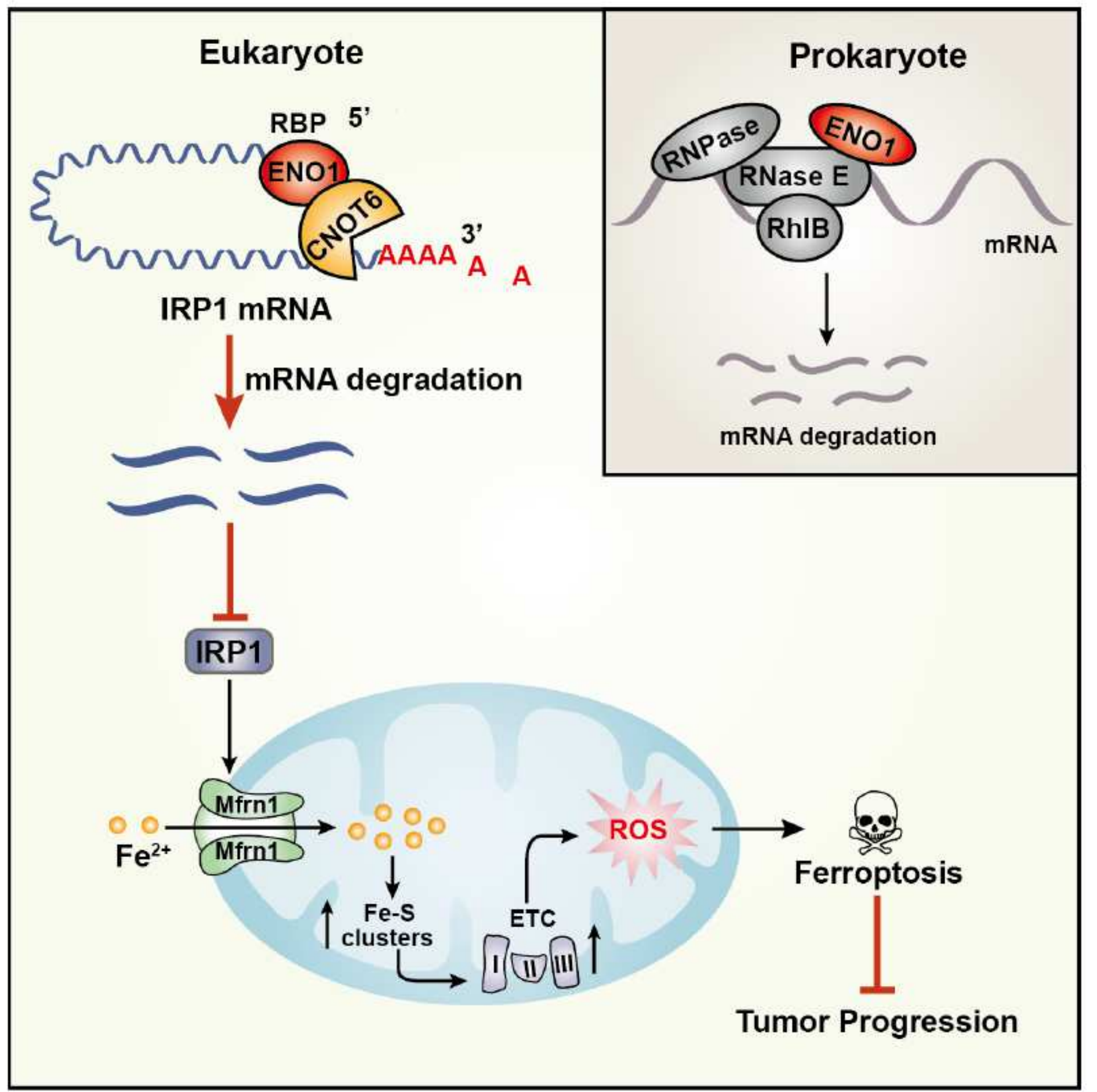

Figure 6 
A schematic model describing the EN01/IRP1/Mfrn1 axis in regulating ferroptosis. A schematic model shows that ENO1, as an RNA-binding protein, recruits CNOT6 to accelerate the mRNA degradation of iron regulatory protein 1 (IRP1) in HCC cells. Consequently, ENO1, via inhibition of IRP1/Mfrn1 axis, suppresses mitochondrial iron-induced ferroptosis.

\section{Supplementary Files}

This is a list of supplementary files associated with this preprint. Click to download.

- ZhangetalSupplementalinformation.pdf 